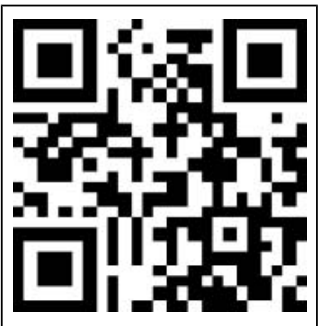

Scan to access more free content
For numbered affiliations see end of article

\section{Correspondence to Jonathan A Drezner, Department of Family Medicine, University of Washington, 1959 NE Pacific Street, Box 356390, Seattle, Washington 98195, USA; jdrezner@uw.edu}

Received 7 December 2012 Revised 7 December 2012 Accepted 7 December 2012
To cite: Drezner JA, Ashley $\mathrm{E}$, Baggish AL, et al. Br J Sports Med 2013;47: 137-152.

\title{
Abnormal electrocardiographic findings in athletes: recognising changes suggestive of cardiomyopathy
}

\author{
Jonathan A Drezner, ${ }_{1}^{1}$ Euan Ashley, ${ }^{2}$ Aaron L Baggish, ${ }^{3}$ Mats Börjesson, ${ }^{4}$ \\ Domenico Corrado, ${ }^{5}$ David S Owens, ${ }^{6}$ Akash Patel, ${ }^{7}$ Antonio Pelliccia, ${ }^{8}$ \\ Victoria L Vetter, ${ }^{7}$ Michael J Ackerman, ${ }^{9,10}$ Jeff Anderson, ${ }^{11}$ Chad A Asplund, ${ }^{12}$ \\ Bryan C Cannon, ${ }^{13}$ John DiFiori, ${ }^{14}$ Peter Fischbach, ${ }^{15}$ Victor Froelicher, ${ }^{2}$ \\ Kimberly G Harmon, ${ }^{1}$ Hein Heidbuchel, ${ }^{16}$ Joseph Marek, ${ }^{17}$ Stephen Paul, ${ }^{18}$ \\ Jordan M Prutkin, ${ }^{6}$ Jack C Salerno, ${ }^{19}$ Christian M Schmied, ${ }^{20}$ Sanjay Sharma, ${ }^{21}$ \\ Ricardo Stein, ${ }^{22}$ Mathew Wilson ${ }^{23}$
}

This document was developed in collaboration between the American Medical Society for Sports Medicine (AMSSM), the Section on Sports Cardiology of the European Association for Cardiovascular Prevention and Rehabilitation (EACPR), a registered branch of the European Society of Cardiology (ESC), the FIFA Medical Assessment and Research Center (F-MARC), and the Pediatric \& Congenital Electrophysiology Society (PACES).

\section{ABSTRACT}

Cardiomyopathies are a heterogeneous group of heart muscle diseases and collectively are the leading cause of sudden cardiac death (SCD) in young athletes. The 12-lead ECG is utilised as both a screening and diagnostic tool for detecting conditions associated with SCD. Fundamental to the appropriate evaluation of athletes undergoing ECG is an understanding of the ECG findings that may indicate the presence of an underlying pathological cardiac disorder. This article describes ECG findings present in cardiomyopathies afflicting young athletes and outlines appropriate steps for further evaluation of these ECG abnormalities. The ECG findings defined as abnormal in athletes were established by an international consensus panel of experts in sports cardiology and sports medicine.

\section{INTRODUCTION}

The cardiomyopathies are a diverse group of heart muscle diseases that are defined and subdivided in clinical practice by different structural and functional characteristics. As a family of related diseases, the cardiomyopathies are the leading cause of sudden cardiac death (SCD) in young competitive athletes. ${ }^{1-3}$ Athletes with an underlying cardiomyopathy may present with disease-related symptoms or may be asymptomatic and thus only identified by abnormal testing during pre-participation screening. Although a definitive diagnosis may require extensive evaluation by a cardiovascular specialist, the 12-lead ECG is commonly abnormal among athletes with an underlying cardiomyopathy. Therefore, it is of paramount importance that clinicians responsible for ECG interpretation in athletes be familiar with key findings associated with underlying diseases of the heart muscle. This paper will review the principal ECG findings associated with the most common forms of cardiomyopathy relevant to the care of the young athlete. Initial testing for further evaluation of abnormal ECG findings is also presented.

\section{DISTINGUISHING NORMAL FROM ABNORMAL}

A challenge in the use of ECG for screening or diagnostic evaluations in athletes is the ability to accurately differentiate findings suggestive of a potentially lethal cardiovascular disorder from benign physiological adaptations occurring as the result of regular and sustained intensive training (ie, athlete's heart). Several reports have outlined ECG criteria intended to distinguish normal ECG findings in athletes from ECG abnormalities requiring additional evaluation. ${ }^{4-9}$

On 13-14 February 2012, an international group of experts in sports cardiology and sports medicine convened in Seattle, Washington, to define contemporary standards for ECG interpretation in athletes. The objective of the meeting was to help physicians distinguish normal ECG alterations in athletes from abnormal ECG findings that require additional evaluation for conditions that predispose to SCD. ${ }^{10}$ A review of normal ECG findings in athletes is presented separately. ${ }^{11}$

In this paper, abnormal ECG findings are presented relative to the most common cardiomyopathies associated with SCD in athletes: hypertrophic cardiomyopathy (HCM), arrhythmogenic right ventricular cardiomyopathy (ARVC), dilated cardiomyopathy (DCM) and left-ventricular non-compaction (LVNC). Table 1 summarises a list of abnormal ECG findings unrelated to athletic training that may suggest the presence of an underlying cardiomyopathy and should trigger additional evaluation in an athlete.

\section{HYPERTROPHIC CARDIOMYOPATHY}

HCM is a genetic disease of the heart muscle. It is characterised by ventricular hypertrophy in the absence of a recognisable cause such as aortic valve disease or hypertension. A common pattern of hypertrophy in HCM is an asymmetric septal hypertrophy where the interventricular septum is thicker than the rest of the left ventricle. However, many other patterns of pathological hypertrophy are consistent with HCM such as apical 
Table 1 Abnormal ECG findings suggestive of cardiomyopathy

\begin{tabular}{|c|c|}
\hline Abnormal ECG finding & Definition \\
\hline T wave inversion & $\begin{array}{l}>1 \mathrm{~mm} \text { in depth in two or more leads V2-V6, II and } \\
\text { aVF or I and aVL (excludes III, aVR and V1) }\end{array}$ \\
\hline ST segment depression & $\geq 0.5 \mathrm{~mm}$ in depth in two or more leads \\
\hline Pathological Q waves & $\begin{array}{l}>3 \mathrm{~mm} \text { in depth or }>40 \mathrm{~ms} \text { in duration in two or } \\
\text { more leads (except III and aVR) }\end{array}$ \\
\hline $\begin{array}{l}\text { Complete left bundle } \\
\text { branch block }\end{array}$ & $\begin{array}{l}Q R S \geq 120 \mathrm{~ms} \text {, predominantly negative } \mathrm{QRS} \text { complex } \\
\text { in lead V1 (QS or } \mathrm{rS} \text { ), and upright monophasic } R \\
\text { wave in leads I and V6 }\end{array}$ \\
\hline $\begin{array}{l}\text { Intraventricular } \\
\text { conduction delay }\end{array}$ & Any $\mathrm{QRS}$ duration $\geq 140 \mathrm{~ms}$ \\
\hline Left axis deviation & $-30^{\circ}$ to $-90^{\circ}$ \\
\hline Left atrial enlargement & $\begin{array}{l}\text { Prolonged } P \text { wave duration of }>120 \mathrm{~ms} \text { in leads I or } \\
\text { II with negative portion of the } P \text { wave } \geq 1 \mathrm{~mm} \text { in } \\
\text { depth and } \geq 40 \mathrm{~ms} \text { in duration in lead } V 1\end{array}$ \\
\hline $\begin{array}{l}\text { Right ventricular } \\
\text { hypertrophy pattern }\end{array}$ & $\begin{array}{l}\mathrm{R}-\mathrm{V}_{1}+\mathrm{S}-\mathrm{V}_{5}>10.5 \mathrm{~mm} \text { AND right axis deviation } \\
>120^{\circ}\end{array}$ \\
\hline $\begin{array}{l}\text { Premature ventricular } \\
\text { contractions }\end{array}$ & $\geq 2$ PVCs per $10 \mathrm{~s}$ tracing \\
\hline Ventricular arrhythmias & $\begin{array}{l}\text { Couplets, triplets and non-sustained ventricular } \\
\text { tachycardia }\end{array}$ \\
\hline
\end{tabular}

hypertrophy, concentric hypertrophy and proximal septal hypertrophy. Poor ventricular compliance (diastolic dysfunction) is characteristic, along with microvascular dysfunction which contribute to ischaemia during exercise. Some patients have dynamic left ventricular (LV) outflow tract obstruction caused by the combination of hypertrophy and abnormalities of the mitral valve which leads to systolic anterior motion of the anterior leaflet. However, only about $25 \%$ of patients with HCM have a murmur from LV outflow tract obstruction during resting examination. ${ }^{12}$ Symptoms of HCM include chest pain, syncope and exercise intolerance, but for many persons the disease can be asymptomatic and SCD may be the clinical presentation of the disease. ${ }^{13}$ Fibrosis of the heart muscle is characteristic and may underlie ventricular arrhythmias and sudden death. On histopathological analysis, disorganised cellular architecture with cardiac myocyte disarray is a hallmark feature. ${ }^{12}$

\section{Prevalence}

HCM is among the most common inherited cardiovascular disorders and may occur in 1:500 adults and at equal prevalence in men and women. ${ }^{12}$ However, the reported prevalence of HCM in competitive athletes is apparently lower, approximately 1 in 1000 to 1 in 1500 athletes. ${ }^{3}{ }^{14} \mathrm{HCM}$ is inherited primarily as autosomal dominant with variable penetrance, and morphological expression of HCM may appear in childhood but typically develops in early adolescence through young adulthood. This may contribute to the lower prevalence of HCM found in younger athletes.

\section{Contribution as a cause of SCD}

In most case series, HCM is among the most common causes of SCD in young athletes. In the USA, HCM accounts for approximately one-third of identified causes of SCD in athletes, and in the UK HCM represents $11 \%$ of cases. ${ }^{1}{ }^{15} \mathrm{HCM}$ is a less common cause of sudden death in other populations. In US military personnel, HCM accounted for only $6 \%$ of SCD, and in the US general population (less than 35 years old) only 5\% of cases of sudden cardiac arrest were attributed to HCM. ${ }^{16} 17$

\section{Diagnostic criteria}

HCM can be diagnosed by ECG in combination with echocardiography or cardiac MRI. An LV wall thickness of $1.5 \mathrm{~cm}$ or greater is normally required to make the diagnosis, but marked asymmetry with lower absolute wall thickness measurement is also compatible with HCM. The upper limit of normal wall thickness in most echocardiography laboratories is $1.2 \mathrm{~cm}$. A 'grey area' is defined between 1.2 and $1.5 \mathrm{~cm}$. In borderline cases, other features favouring a diagnosis of HCM include impaired diastolic function, small LV cavity size, LV wall thickness asymmetry, mitral valve pathology (leaflet redundancy and systolic anterior motion) and the presence of myocardial fibrosis (late gadolinium enhancement) on cardiac MRI. ${ }^{18}$

\section{Abnormal ECG findings in HCM}

Over $90 \%$ of patients with HCM will have an abnormal ECG. ${ }^{19-21}$ ECG abnormalities include $\mathrm{T}$ wave inversion (TWI), ST segment depression, pathological Q waves, conduction delay, left-axis deviation (LAD) and left atrial enlargement (LAE).

T wave inversion

TWI in the lateral or inferolateral leads is seen commonly in HCM (figures 1-3). In a series of asymptomatic patients $\leq 35$ years old with HCM confirmed by cardiac MRI, $62 \%$ exhibited TWI. ${ }^{21}$ Similarly, in patients with a positive HCM genetic test and overt morphological HCM, 54\% demonstrate TWI. ${ }^{22}$ In black patients with HCM, TWI occurs more commonly in the lateral leads (77\%) and less frequently in the inferior leads (2\%). ${ }^{23}$ Abnormal TWI is defined as $>1 \mathrm{~mm}$ in depth in two or more leads V2-V6, II and aVF, or I and aVL (excludes leads III, aVR and V1). Deep TWI in the mid-precordial to lateral precordial leads (V4-V6) should raise the possibility of apical HCM.

In healthy athletes, TWI in the lateral or inferior leads is uncommon. TWI beyond V2 is a rare abnormality found in only $0.1 \%$ of Caucasian adolescent athletes older than 16 years. $^{24}$ In a college athletic population of mixed ethnicity, TWI in the lateral or inferolateral leads is reported in $2 \%$ of athletes. ${ }^{25}$ In Caucasian elite athletes, the prevalence of TWI in the lateral or inferior leads is also about $2 \% .{ }^{26}$ However, TWI is more common in black athletes of African-Caribbean descent (hereto referred to as 'black/African' athletes). TWI in the lateral or inferior leads is reported in $8-10 \%$ of black/African athletes. $^{23} 27$

\section{Repolarisation variant in black/African athletes}

TWI in the anterior precordial leads should be distinguished from TWI in the lateral or inferior leads in black/African athletes. TWI in the anterior precordial leads may be part of a normal variant pattern of repolarisation in black/African athletes consisting of convex ('dome' shaped) ST segment elevation followed by TWI in V1-V4 (figure 4). On the basis of current data, TWI preceded by ST segment elevation are present in the anterior precordial leads in up to $13 \%$ of black/African athletes and do not require further assessment in the absence of symptoms, positive family history or abnormal physical examination. $^{23}{ }^{27}$ However, TWI in the lateral or inferolateral leads (V5-V6, I and aVL, II and aVF), regardless of ethnicity, is considered abnormal and requires additional testing to rule out HCM (figures 1-3). 


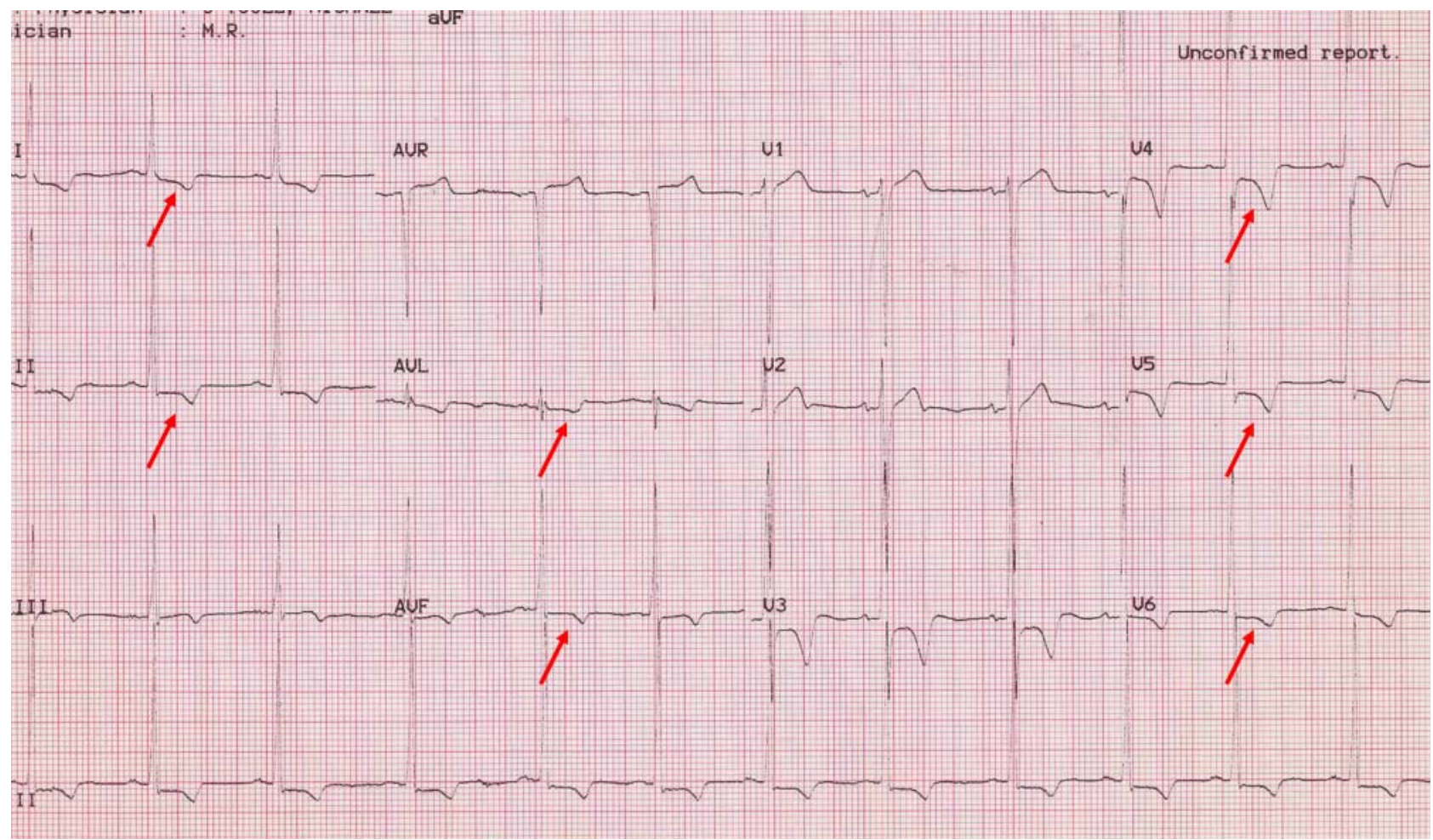

Figure 1 Abnormal ECG in a patient with hypertrophic cardiomyopathy. Note the T wave inversion and ST depression in the inferolateral leads (arrows). This figure is only reproduced in colour in the online version.

\section{Juvenile pattern of TWI}

TWI in the anterior precordial leads in younger, prepubertal athletes often reflects a persistent juvenile pattern and requires careful interpretation. In Caucasian adolescent athletes, anterior precordial TWI extending beyond V2 was present in $1.2 \%$ of athletes $<16$ years but only $0.1 \%$ of athletes $\geq 16$ years. ${ }^{24}$ In a study of Italian adolescent athletes, incomplete pubertal development was an independent predictor for right precordial
TWI. ${ }^{28}$ The prevalence of right precordial TWI decreased significantly with increasing age, $8.4 \%$ in children $<14$ years of age versus $1.7 \%$ in those $\geq 14$ years. ${ }^{28}$

\section{Biphasic T waves}

Biphasic Twaves create a challenge and currently there is no consensus regarding the definition of TWI when a large positive deflection precedes a negative portion below the isoelectric line. If

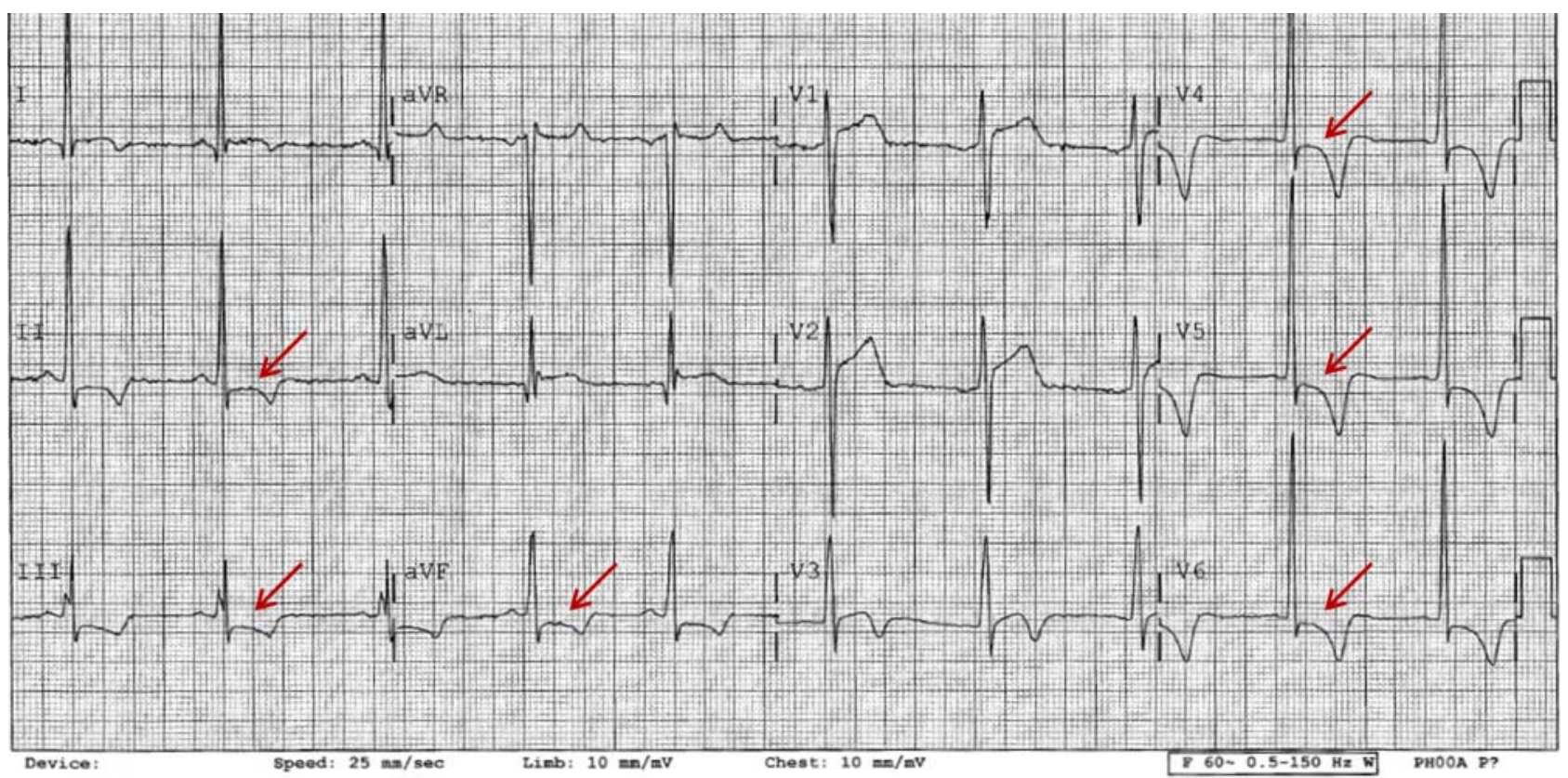

Figure 2 Markedly abnormal ECG in a patient with hypertrophic cardiomyopathy. Note deep T wave inversion and ST depression in the inferolateral leads. This figure is only reproduced in colour in the online version. 


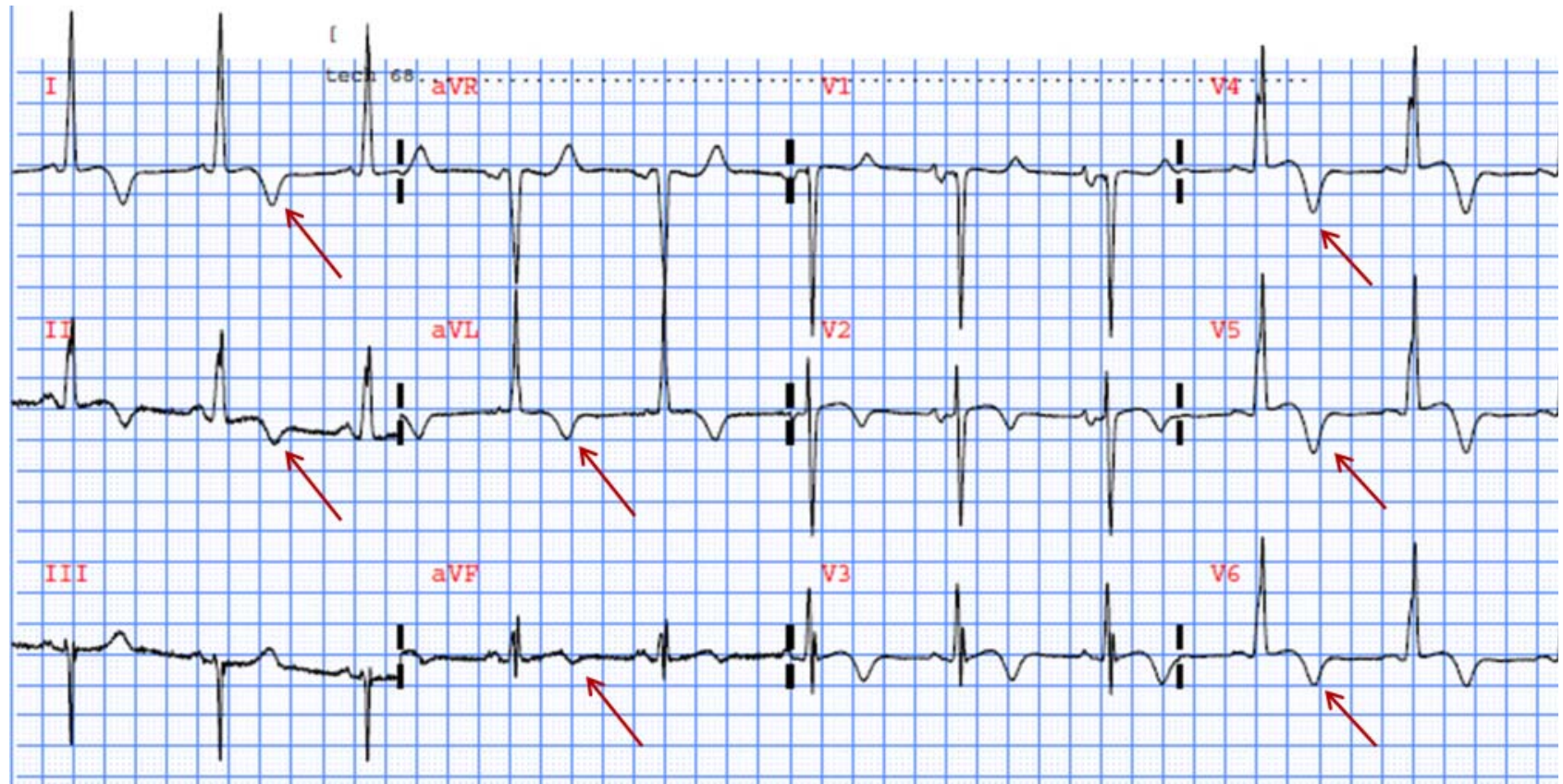

Figure 3 Markedly abnormal ECG in a patient with hypertrophic cardiomyopathy. Note the deep T wave inversions in the inferolateral leads (V4V6, I and aVL, II and aVF). This ECG pattern may represent apical hypertrophic cardiomyopathy which is not adequately evaluated by echocardiography. Cardiac MRI is recommended. This figure is only reproduced in colour in the online version.

the negative portion of the $\mathrm{T}$ wave is $>1 \mathrm{~mm}$ in depth in two or more leads (excluding leads III, aVR, and V1), it is reasonable to consider this pattern as abnormal until more data are obtained.

\section{ST segment depression}

ST segment depression is a common abnormality in HCM but extremely rare in otherwise healthy athletes, making it a concerning indicator of disease if identified on an athlete's ECG. ST segment depression is reported in $46-50 \%$ of patients with HCM, but in $<1 \%$ of apparently healthy athletes or adolescents undergoing ECG screening. ${ }^{9}$ 22-25 Any degree of ST depression beyond $0.5 \mathrm{~mm}$ in two or more leads is significant and requires further investigation for cardiomyopathy (figures 1 and 2).

\section{Pathological Q waves}

$\mathrm{Q}$ waves have been defined in different ways in different populations. In patients with overt HCM, pathological Q waves are

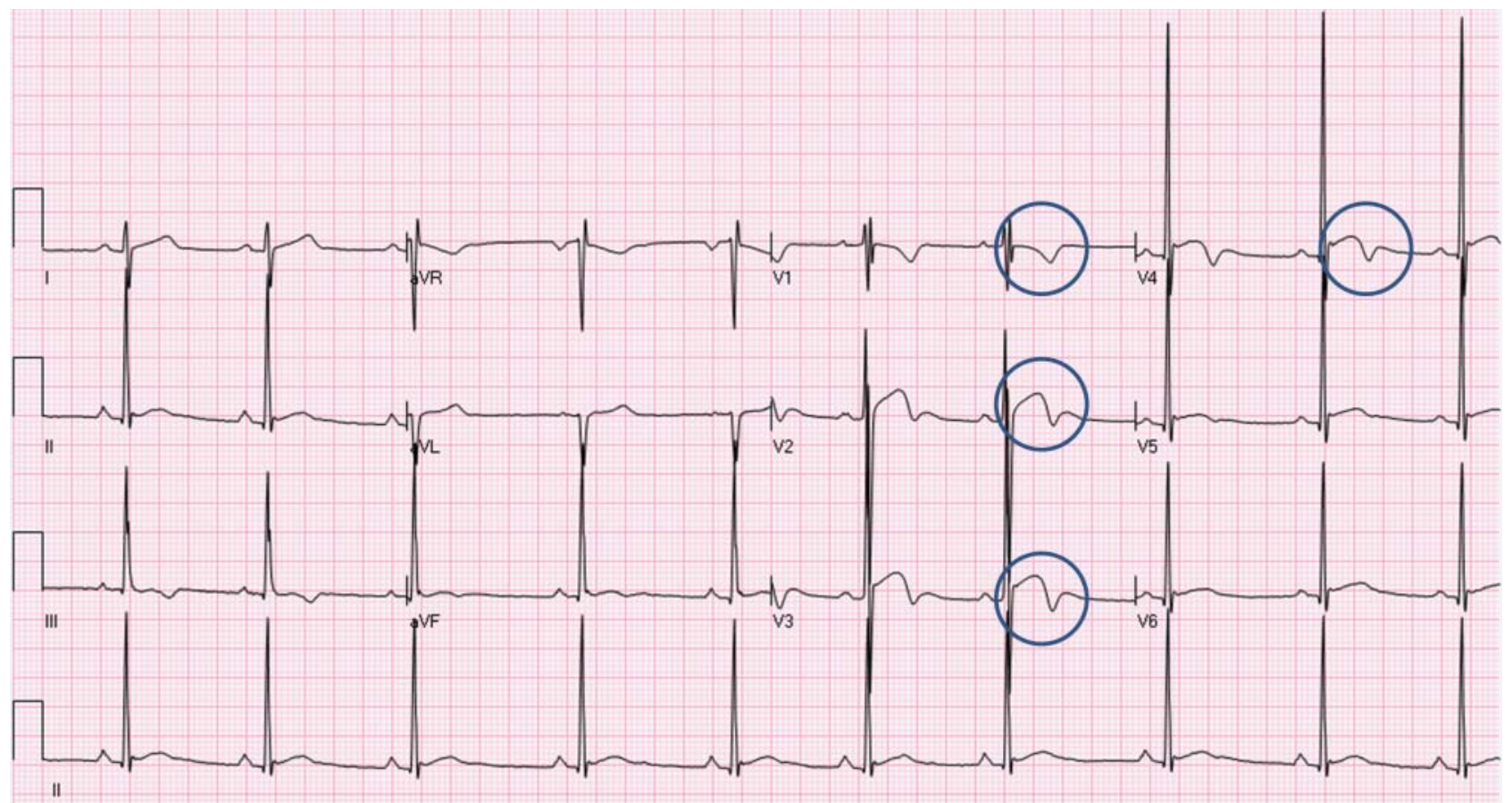

Figure 4 ECG from a 17-year-old black/African soccer player demonstrating 'domed' ST elevation followed by T wave inversion in leads V1-V4 (circles). This is a normal repolarisation pattern in black/African athletes and does not require further investigation in asymptomatic athletes. Note the T wave inversion does not extend to the lateral leads beyond V4. This figure is only reproduced in colour in the online version. 
reported in $32-42 \%$ of patients. ${ }^{19}{ }^{22}$ In one series of asymptomatic patients with HCM, 42\% demonstrated pathological Q waves. $^{21}$ The consensus of this group is to define $\mathrm{Q}$ waves for $\mathrm{HCM}$ as $>3 \mathrm{~mm}$ in depth or $>40 \mathrm{~ms}$ in duration in at least two leads (excluding leads III and aVR; figure 5). This detects HCM with a sensitivity of $35 \%$ and a specificity of $95 \%$ in patients with preclinical HCM based on molecular genetic diagnosis. ${ }^{29}$

\section{Intraventricular conduction delay}

Left bundle branch block (LBBB) is an abnormal finding detected in $2 \%$ of patients with HCM but not reported in screening populations of athletes or adolescents. $9{ }^{22} 25$ LBBB pattern with a QRS duration of $120 \mathrm{~ms}$ or greater should prompt further evaluation (figure 6). Right bundle branch block (RBBB) is found more commonly in HCM than in athletes but the frequency of incomplete and complete RBBB in athletes is felt to limit its differentiating value. ${ }^{23}{ }^{30}$ The significance of a non-specific intraventricular conduction delay (IVCD) with normal QRS morphology is uncertain. However, marked nonspecific IVCD $>140 \mathrm{~ms}$ is considered abnormal and should prompt further evaluation.

\section{Left axis deviation}

LAD, defined as $-30^{\circ}$ to $-90^{\circ}$, is present in almost $12 \%$ of HCM patients but less than 1\% of athletes (figure 7). ${ }^{9} 2325$ LAD can be a secondary marker for pathological LV hypertrophy (LVH) and if present warrants additional evaluation.

\section{Left atrial enlargement}

ECG findings suggestive of LAE have been defined in different ways. Overall, LAE on ECG is present in approximately 10$21 \%$ of HCM patients but has been reported in up to $44 \%$ of black patients with HCM (figure 8). ${ }^{21-23}$ LAE is defined as a prolonged $\mathrm{P}$ wave duration of $>120 \mathrm{~ms}$ in leads I or II with negative portion of the $P$ wave $\geq 1 \mathrm{~mm}$ in depth and $\geq 40 \mathrm{~ms}$ in duration in lead V1. LAE on ECG is an uncommon finding in athletes and should prompt additional investigation.
Increased QRS voltage in athletes and HCM

The isolated presence of high QRS voltages fulfilling voltage criterion for $\mathrm{LVH}$ is regarded as a normal finding in athletes related to physiological increases in cardiac chamber size and/or wall thickness and does not in itself require additional evaluation. ${ }^{5}$ As expected, voltage criteria for LVH also are commonly identified in individuals with HCM. However, the presence of isolated increased QRS voltage in the absence of other ECG abnormalities is uncommon and present in $<2 \%$ of individuals with the disease. ${ }^{19}$ Several studies have evaluated athletes and young adults with isolated increased QRS voltage using echocardiography or cardiac MRI and none had HCM. $^{24} 26$ 31-33 Therefore, isolated increased QRS voltage on the ECG in the absence of other abnormalities in an asymptomatic athlete with a negative family history is not a reliable indicator of HCM and does not require further evaluation. Co-existing ECG abnormalities such as TWI, ST segment depression, pathological Q waves, IVCD, LAD or LAE should be investigated by additional testing.

\section{Evaluation of suspected HCM}

If HCM is suspected based on ECG abnormalities, evaluation of LV morphology and function is required. ${ }^{34}$ Echocardiography provides assessment of LV cavity size and wall thickness, systolic and diastolic function and valvular structure and function, and is the first test of choice under most circumstances. HCM can be diagnosed when wall thickness is $\geq 1.5 \mathrm{~cm}$ with normal or small LV cavity size in the absence of other causes capable of causing myocardial hypertrophy. Diastolic dysfunction, mitral valve pathology and LV outflow tracts obstruction are other findings that support the diagnosis of HCM.

However, echocardiographic quality is variable based on numerous factors, including operator proficiency and patient acoustic windows, and may have limited ability to detect hypertrophy of the anterolateral LV wall and apex (figure 9). ${ }^{35}$ Cardiac MRI provides superior assessment of myocardial hypertrophy and may demonstrate late gadolinium enhancement which is a non-specific marker suggesting myocardial fibrosis. Cardiac MRI should be considered when echocardiography is insufficient to assess all myocardial segments or when

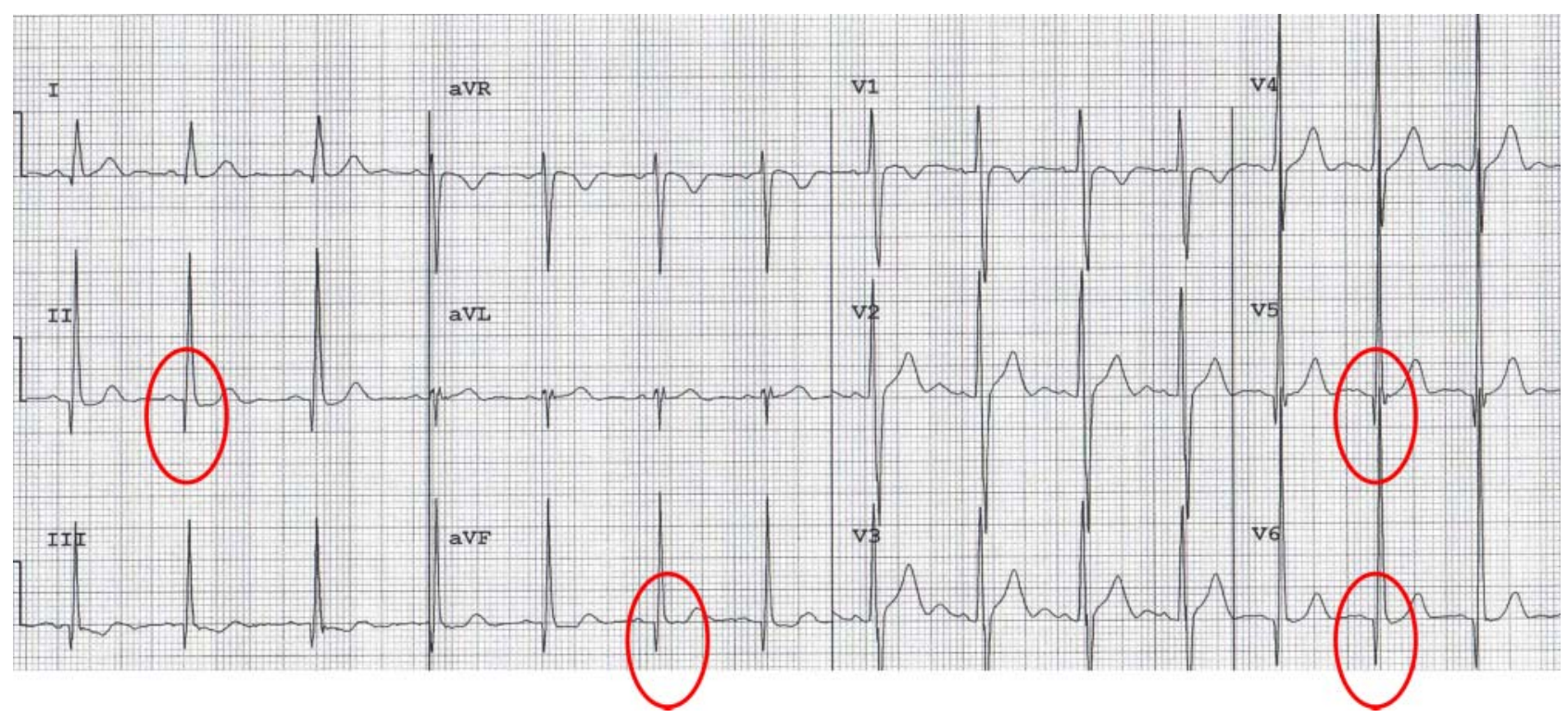

Figure 5 Abnormal ECG in a patient with hypertrophic cardiomyopathy. Note the abnormal Q waves (>3 mm in depth) in V5-V6, II and aVF. This figure is only reproduced in colour in the online version. 


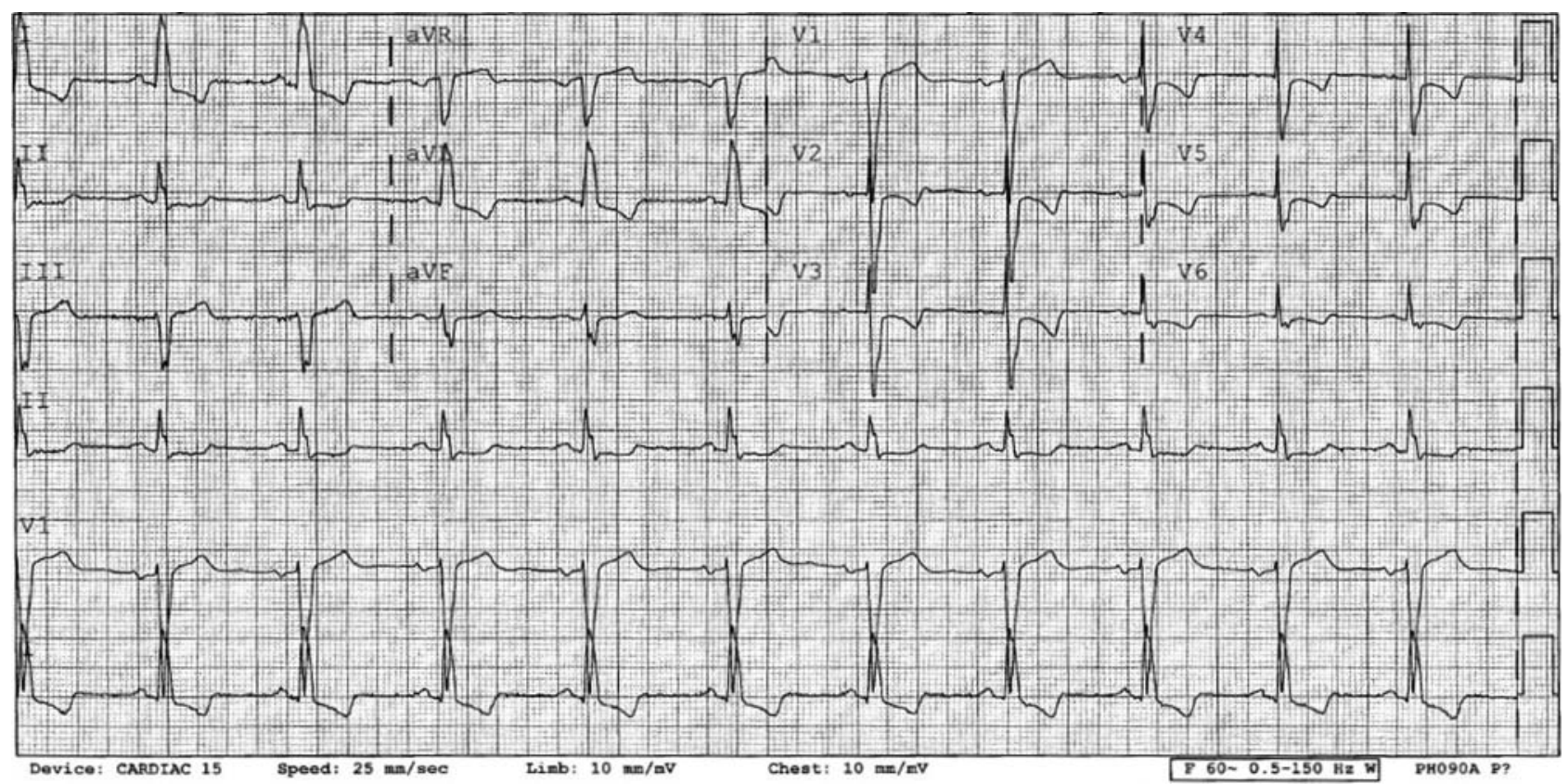

Figure 6 Abnormal ECG in a patient with hypertrophic cardiomyopathy showing complete left bundle branch block (QRS $\geq 120$ ms with predominantly negative QRS complex in lead V1).

myocardial hypertrophy falls into the 'grey zone' between 1.2 and $1.5 \mathrm{~cm}$. Cardiac MRI is recommended for markedly abnormal ECGs suggestive of apical HCM, specifically ECGs with deep TWI and/or ST depression in the inferolateral leads (V4V6, I, aVL, II and aVF), in which echocardiography often does not provide an adequate assessment of the LV apex or inferior septum (figures 1-3).

A common clinical dilemma is the detection of myocardial hypertrophy in an athlete in which the hypertrophy may be due to physiological adaptation to exercise. Differentiating athlete's heart from HCM requires careful clinical evaluation by an experienced provider. ${ }^{36-38}$ Cardiac MRI, cardiopulmonary exercise testing and Holter monitoring should be considered. Findings suggestive of HCM include the presence of unusual patterns of hypertrophy with substantial differences in wall thickness of the LV segments, normal or reduced LV cavity size, extreme LAE, diastolic dysfunction, family history of HCM or
SCD, below normal peak oxygen consumption (peak $\mathrm{VO}_{2}$ ), and the presence of ventricular arrhythmias. When diagnostic uncertainty remains, genetic testing and/or a period of deconditioning followed by reassessment to document regression (or lack thereof) of exercise-induced LVH may be considered.

\section{Long-term follow-up of markedly abnormal ECGs}

Highly trained athletes occasionally present markedly abnormal ECG patterns instinctively suggesting the presence of a cardiomyopathy. ${ }^{39} 40$ Such abnormal ECGs raise the question of differentiating between the initial, subtle expression of cardiac disease or the extreme but innocent ECG expression of the 'athlete's heart'. 264142

Investigators researched the clinical outcomes of 81 athletes presenting initially with markedly abnormal repolarisation patterns in the absence of detectable cardiac abnormalities. ${ }^{43}$ After an average 9-year follow-up, a new diagnosis of
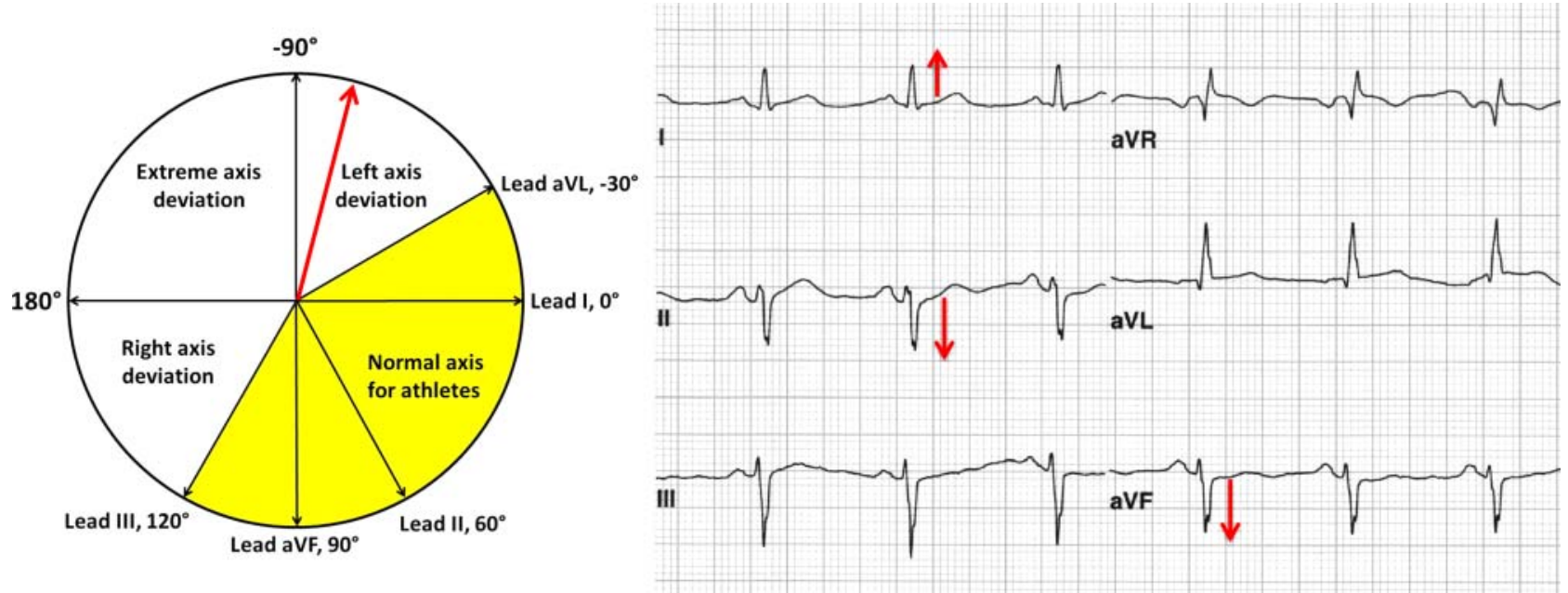

Figure 7 ECG demonstrates abnormal left-axis deviation defined as frontal plane QRS axis of less than $-30^{\circ}$. The QRS is positive in lead I and negative in $\mathrm{aVF}$ and lead II. The QRS axis shown here is about $-70^{\circ}$. This figure is only reproduced in colour in the online version. 


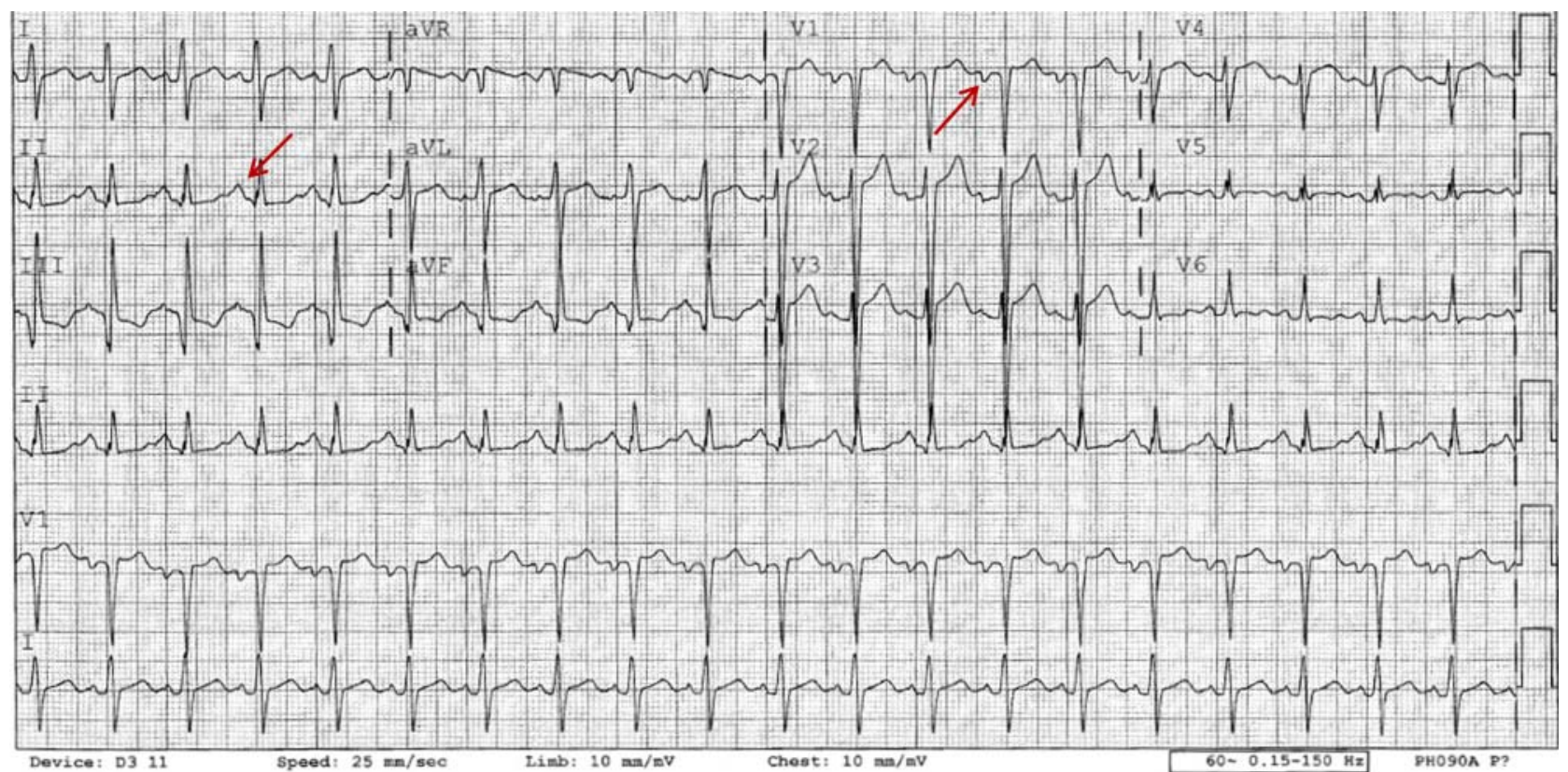

Figure 8 Abnormal ECG in a patient with hypertrophic cardiomyopathy showing left atrial enlargement, defined as a prolonged $\mathrm{P}$ wave duration $>120 \mathrm{~ms}$ in leads I or II with negative portion of the $\mathrm{P}$ wave $\geq 1 \mathrm{~mm}$ in depth and $\geq 40 \mathrm{~ms}$ duration in lead V1. This figure is only reproduced in colour in the online version.

cardiomyopathy was made in five athletes (6\%), including three with HCM, one with ARVC and one with DCM. ${ }^{43}$ Two athletes experienced adverse events $(0.3 \%$ per year $)$, including one cardiac arrest from HCM and one sudden death related to ARVC. ${ }^{43}$ Markedly abnormal ECGs, therefore, may represent the initial expression of cardiomyopathy, preceding by many years the phenotypic or morphological expression of structural heart disease.
Athletes presenting with distinctly abnormal ECGs (ie, deep TWI in the lateral leads) and no evidence of structural heart disease after a thorough work-up may be permitted to participate in competitive athletics. However, these athletes should undergo serial clinical evaluation on an annual basis, even in the absence of symptoms, including repeat imaging tests such as echocardiography and/or cardiac MRI to evaluate for the development of cardiomyopathy.
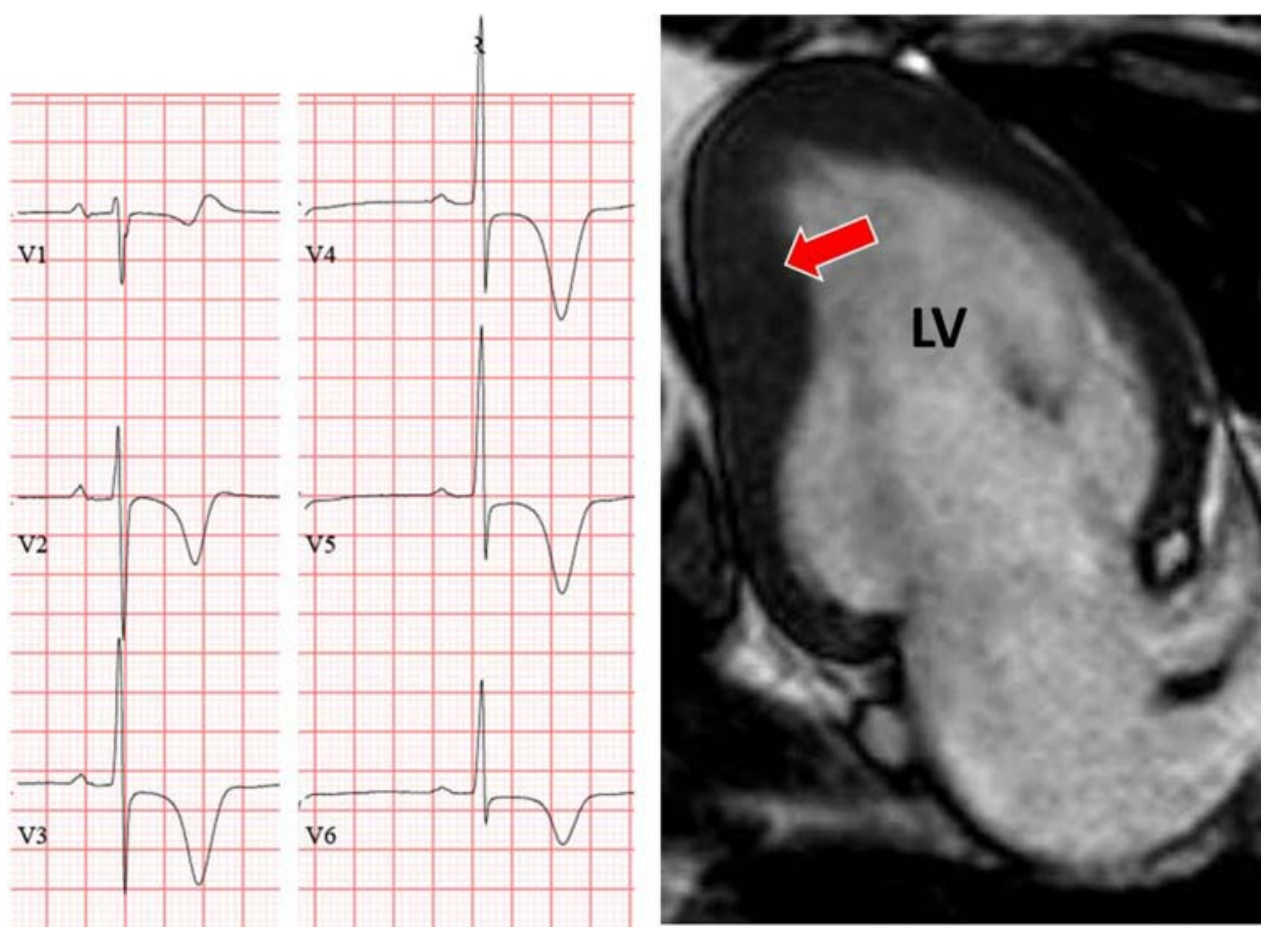

Figure 9 ECG (left panel) and cardiac MRI (right panel) of apical hypertrophic cardiomyopathy. Deep T wave inversions across the precordial leads are a characteristic ECG finding. The region of hypertrophy (red arrow) is isolated to the left ventricular (LV) apex, which can be challenging to detect by echocardiography. This figure is only reproduced in colour in the online version. 


\section{ARRHYTHMOGENIC RIGHT VENTRICULAR CARDIOMYOPATHY}

ARVC is an inherited heart muscle disease characterised by fibro-fatty replacement of right ventricular myocardium and corollary life-threatening ventricular arrhythmias or SCD, mostly in young people and athletes. Progressive dilation/dysfunction predominantly involves the right ventricle with involvement of the left ventricle in late-stage disease. Variants with predominantly $\mathrm{LV}$ involvement are described in about $10 \%$ of patients (hence the alternative term of arrhythmogenic cardiomyopathy). Mutations in the desmosomal genes account for approximately $50 \%$ of ARVC cases. $^{44}{ }^{45}$ In addition, there is emerging evidence that intense endurance sports may lead to a similar phenotype (with similar prognosis) in the absence of desmosomal mutations, so-called exercise-induced ARVC, which may be the result of increased RV wall stress during exercise. ${ }^{46-48}$ The prevalence of familial ARVC is estimated at $1: 2000-1$ : 5000 persons. $^{44} 45$

\section{Contribution as a cause of SCD}

According to data from the Veneto region of Italy where postmortem investigation of young sudden death victims is performed systematically, ARVC is a leading cause of sport-related sudden death accounting for approximately one-fourth of fatalities in young competitive athletes. ${ }^{3}$ Data from the USA, notably without a mandatory registry for SCD in athletes, suggest that ARVC is a less common cause of SCD. ${ }^{1}$

\section{Diagnostic criteria}

The original (1994) and the revised (2010) Task Force Criteria for diagnosis of ARVC are based on major and minor criteria encompassing familial/genetic, ECG, arrhythmic, morphofunctional ventricular and histopathological features. ${ }^{49}$ The diagnosis is fulfilled in the presence of two major criteria, one major plus two minor criteria, or four minor criteria from different groups. $^{49}$

\section{Abnormal ECG findings in ARVC}

Over $80 \%$ of patients with ARVC will have an abnormal ECG. ${ }^{45} 5051$ ECG abnormalities include TWI in the anterior precordial leads, epsilon waves, delayed $S$ wave upstroke, low- voltage in limb leads and premature ventricular beats with an LBBB morphology and superior axis. If there is primarily LV involvement, the TWI involves the lateral precordial leads and the premature ventricular beats can have an RBBB morphology.

T wave inversion

TWI in the anterior precordial leads (V1-V3/V4) is present in approximately $85 \%$ of patients with ARVC in the absence of RBBB (figures 10 and 11). ${ }^{50}$ TWI occasionally extends to the left precordial leads V5-V6 or inferior limb leads II, III and aVF. TWI in V1-V3 or beyond in individuals $>14$ years of age (in the absence of complete RBBB) represent a major diagnostic criterion for ARVC, while TWI confined to just leads V1 and $\mathrm{V} 2$ in individuals $>14$ years of age (in the absence of complete $\mathrm{RBBB})$ represents a minor diagnostic criterion. ${ }^{49}$ In Italian children $\geq 14$ years with TWI in the anterior precordial leads beyond V2 (ie, V3 or V4), 3 of 26 (11\%) fulfilled diagnostic criteria for ARVC (1 definitive, 2 borderline). ${ }^{28}$

In the presence of complete RBBB, right precordial (V1-V3) TWI is more likely secondary to RBBB rather than a sign of underlying ARVC. TWI extending beyond V3 is uncommon in patients with RBBB and represents a minor diagnostic criterion for ARVC. ${ }^{49}$

Thus, TWI involving at least two consecutive precordial leads, excluding V1, should prompt further investigation in the athlete.

\section{Epsilon waves}

Epsilon waves are defined as distinct low-amplitude potentials localised at the end of the QRS complex. Epsilon waves are challenging to detect and appear as a small negative deflection just beyond the QRS in V1-V3 (figure 10). The presence of epsilon waves in the right precordial leads $\mathrm{V} 1-\mathrm{V} 3$ represents a major diagnostic criterion for ARVC. ${ }^{49}$

\section{Delayed S wave upstroke}

Delayed S wave upstroke of $>55 \mathrm{~ms}$ in leads V1-V3 in the absence of complete RBBB represents a minor diagnostic criterion for ARVC (figure 11). ${ }^{49}$ This feature is most commonly observed among ARVC patients with mild QRS prolongation $(100-120 \mathrm{~ms})$. The $\mathrm{S}$ wave upstroke is measured from the nadir
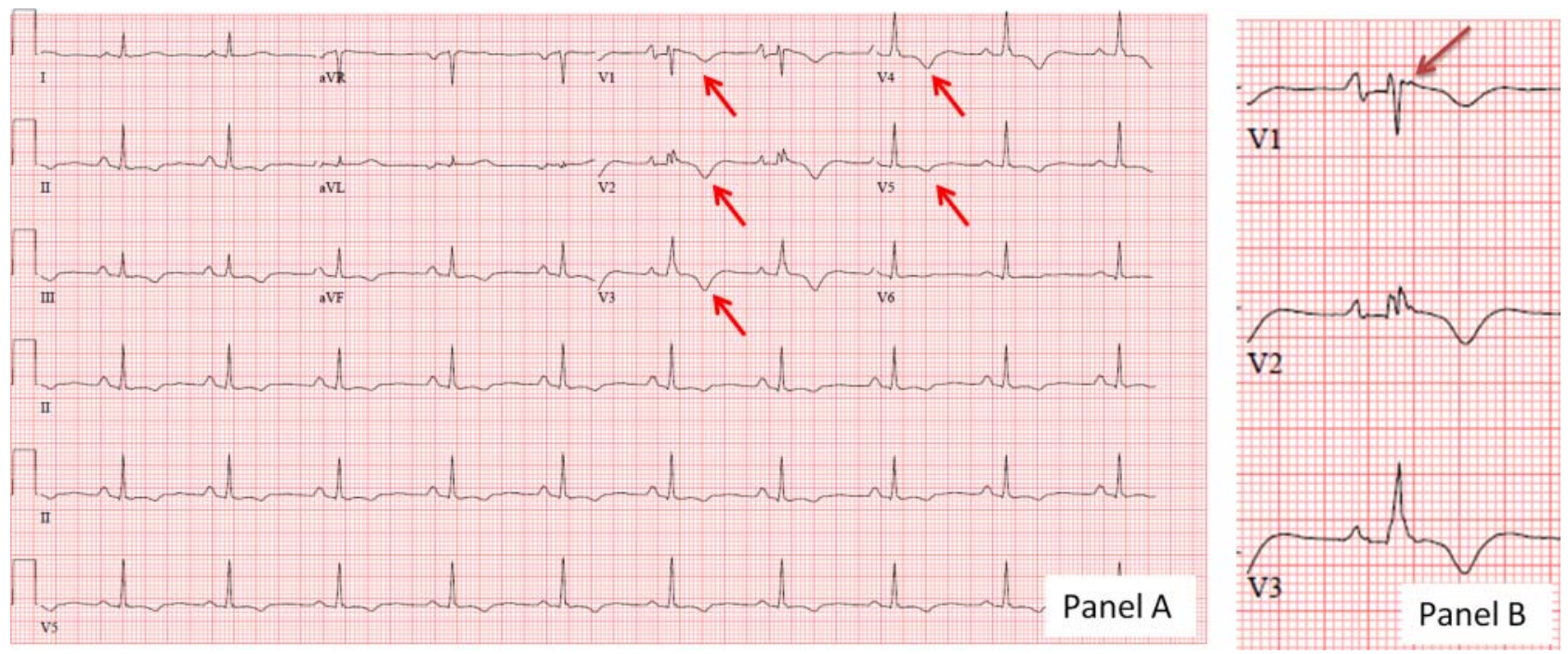

Figure 10 ECG from a patient with arrhythmogenic right ventricular cardiomyopathy. (A) Inverted T waves in leads V1-V5. (B) A subtle epsilon wave with notching in V1 at the terminal portion of the QRS complex. This figure is only reproduced in colour in the online version. 

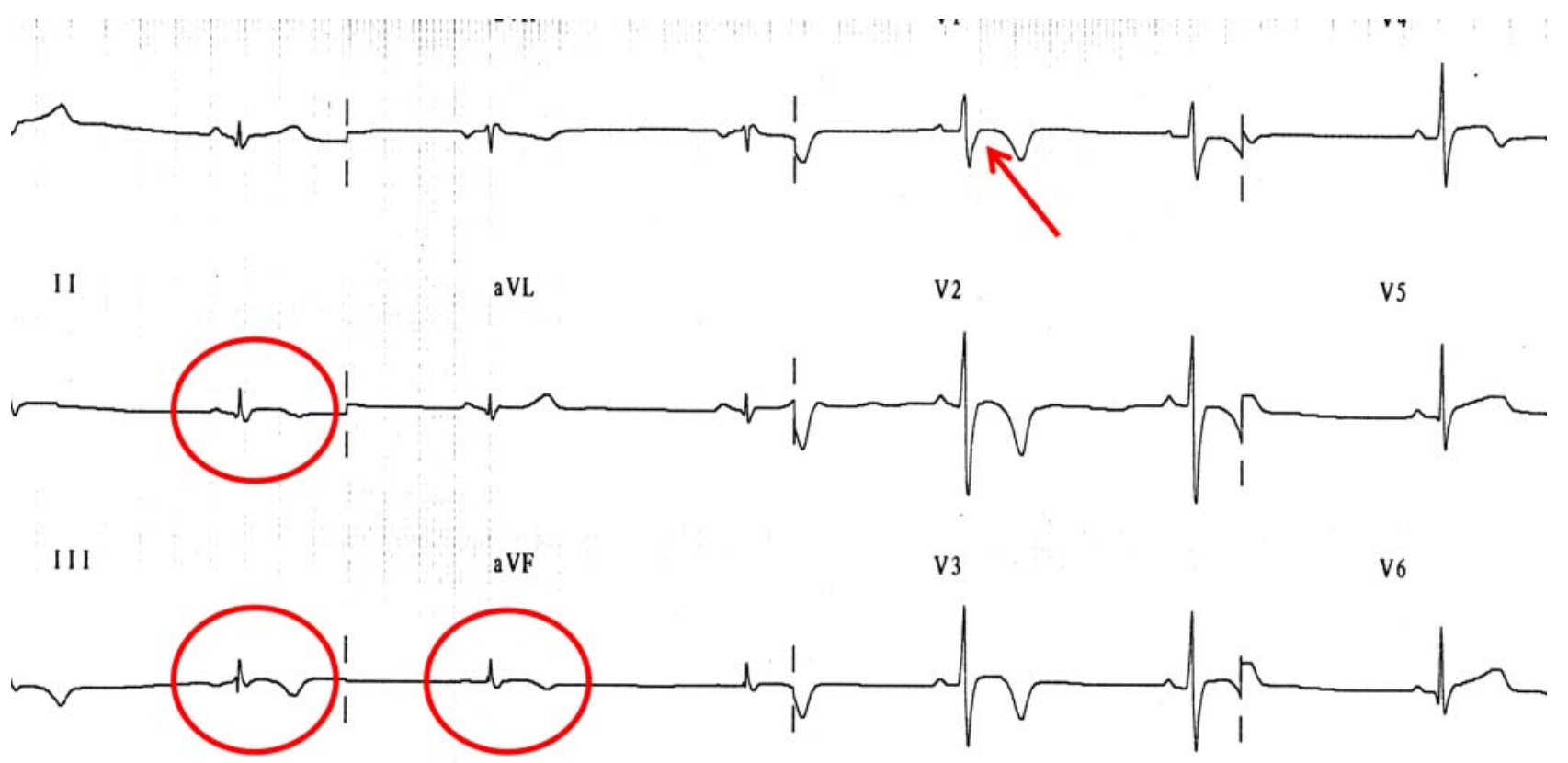

II

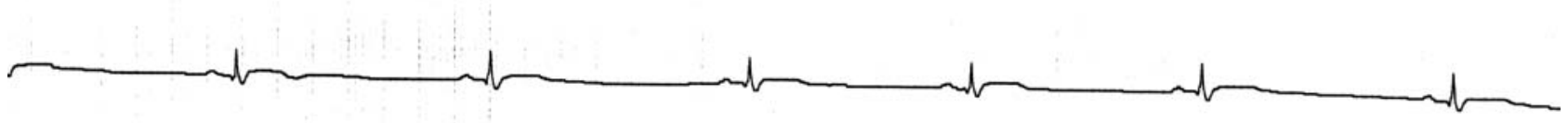

Figure 11 ECG from a patient with arrhythmogenic right ventricular cardiomyopathy showing delayed S wave upstroke in V1 (arrow), low voltages in limb leads $<5 \mathrm{~mm}$ (circles), and inverted T waves in anterior precordial leads (V1-V4) and inferior leads (III and aVF). This figure is only reproduced in colour in the online version.

of the $S$ wave to the end of the QRS (including epsilon wave if present). Prolonged $S$ wave upstroke may be present in up to $95 \%$ of patients with ARVC in the absence of RBBB. ${ }^{50}$

Low voltage in limb leads

Low voltage in limb leads, defined as a QRS amplitude $\leq 5 \mathrm{~mm}$ in each of the limb leads (I, II and III), also can be suggestive of ARVC (figure 11).

\section{Premature ventricular contractions}

Premature ventricular contractions (PVCs) originating from the right ventricle typically show an LBBB pattern with a predominantly negative QRS complex in V1. On the basis of the QRS axis in the limb leads the origin of the PVC can be further suggested. PVCs with LBBB morphology and an inferior axis (positive in the inferior leads) originate from the right ventricular outflow tract consistent with idiopathic right ventricular outflow tract arrhythmia which is a benign condition, non-familial and not associated with structural ventricular abnormalities. PVCs with an LBBB morphology and superior axis (negative in the inferior leads) originate from the right ventricular free wall or apex and are more suggestive of ARVC (figure 12).

\section{Evaluation of suspected ARVC}

Disease expression in ARVC is variable, and clinical manifestations vary with age and stage of disease. ${ }^{49}$ Similarly, the extent of ECG abnormalities are associated with the severity of disease. ${ }^{51}$ In patients with diagnosed ARVC ('overt stage') or known desmosome mutation positive subjects, 95\% have an abnormal ECG marked by abnormal TWI, a prolonged $\mathrm{S}$ wave upstroke in the anterior precordial leads (V1-V3), and/or an epsilon wave. ${ }^{50-52}$ However, in cardiac screening, physicians may encounter asymptomatic athletes in the early 'concealed stage' of the disease, showing less pronounced ECG changes.

The extent of evaluation is dependent on the specific ECG findings suggestive of ARVC and will be more extensive in the presence of warning symptoms or significant family history. A combination of tests is needed to effectively make the diagnosis or to rule out ARVC. Echocardiography, ambulatory ECG (Holter) monitoring, signal-averaged ECG (SAECG), and ventricular angiography provided optimal evaluation, while cardiac MRI (false-positives) and biopsy (low-sensitivity) were considered less useful for diagnosis in suspected ARVC. ${ }^{53} 54$

The evaluation of major diagnostic ECG findings according to the 2010 Task Force criteria of TWI in the right precordial leads (V1-V3) or beyond in ages >14 years (in the absence of complete RBBB) should be extensive. In addition to a comprehensive symptom history, family history and physical examination, evaluation of TWI in V1-V3 or beyond should include echocardiography, Holter monitoring, SAECG, maximal exercise-ECG test and possibly a cardiac MRI. ${ }^{49}$ Isolated epsilon waves in the right precordial leads, a less specific major ECG criteria, still require an echocardiography and ECG monitoring for an arrhythmia (Holter or exercise-ECG test). ${ }^{49}$

Evaluation of minor diagnostic ECG-findings according to the Task Force criteria (without accompanying positive family history or alarming symptoms), may be less extensive. ${ }^{49}$ TWI in V1-V2 may require simply a careful personal and family history and physical examination. 


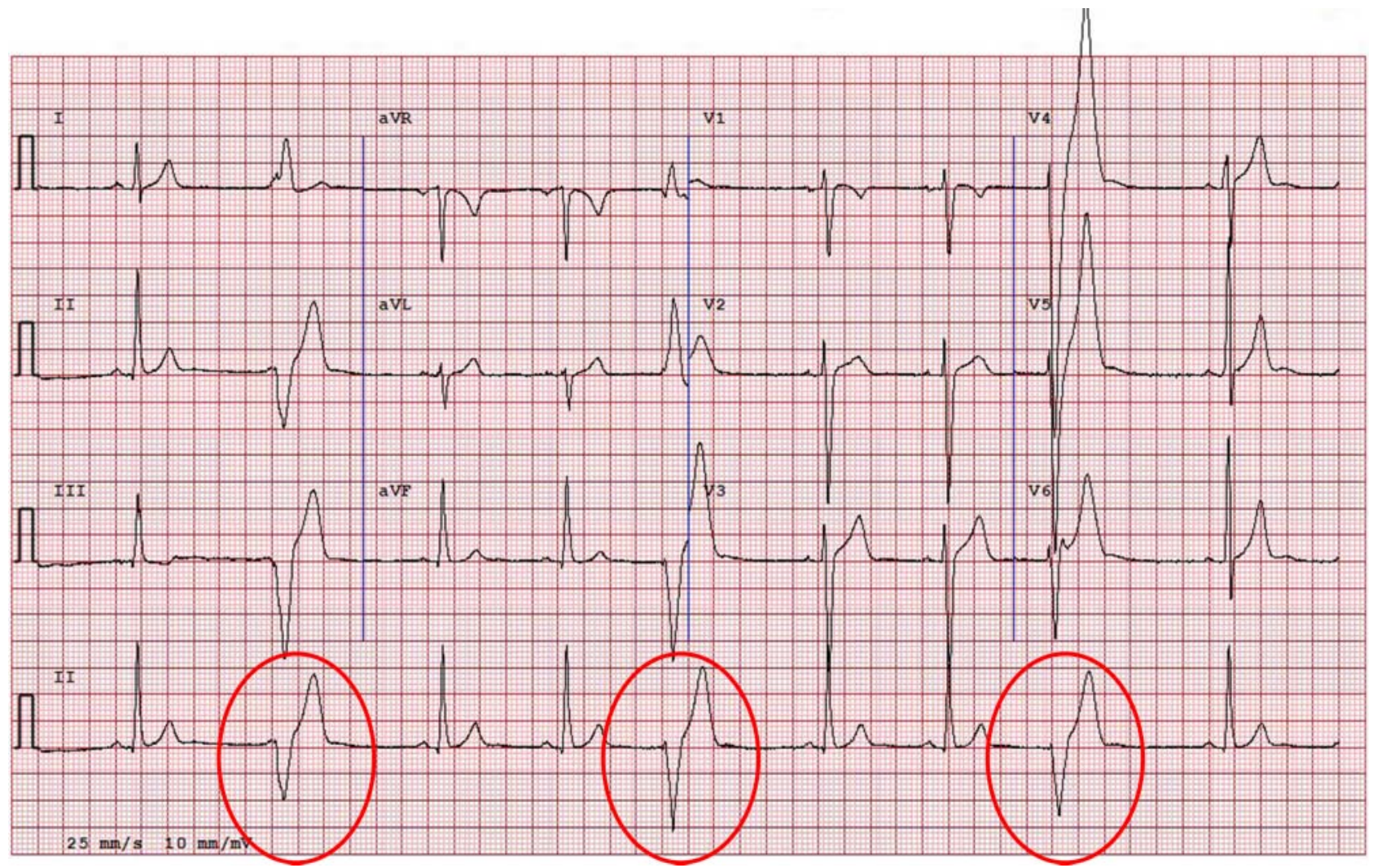

Figure 12 ECG from a patient with arrhythmogenic right ventricular cardiomyopathy. Note multiple premature ventricular complexes with a left bundle branch pattern and superior axis (negative QRS vector in inferior leads). This figure is only reproduced in colour in the online version.

Repolarisation variants in the anterior precordial leads in black/African athletes must be distinguished from pathological repolarisation changes found in ARVC. In ARVC, the ST segment is usually isoelectric prior to TWI, in contrast to the 'domed' ST segment elevation which is the hallmark feature of the normal repolarisation variant in black/African athletes (figure 13). TWI involving at least two consecutive precordial leads from V2 to V6 with an isoelectric ST segment, regardless of ethnicity, requires additional investigation.

\section{DILATED CARDIOMYOPATHY}

DCM is a heart muscle disorder characterised by weakened myocardial contraction, which over time leads to cavity enlargement and eccentric heart muscle hypertrophy. It is a common

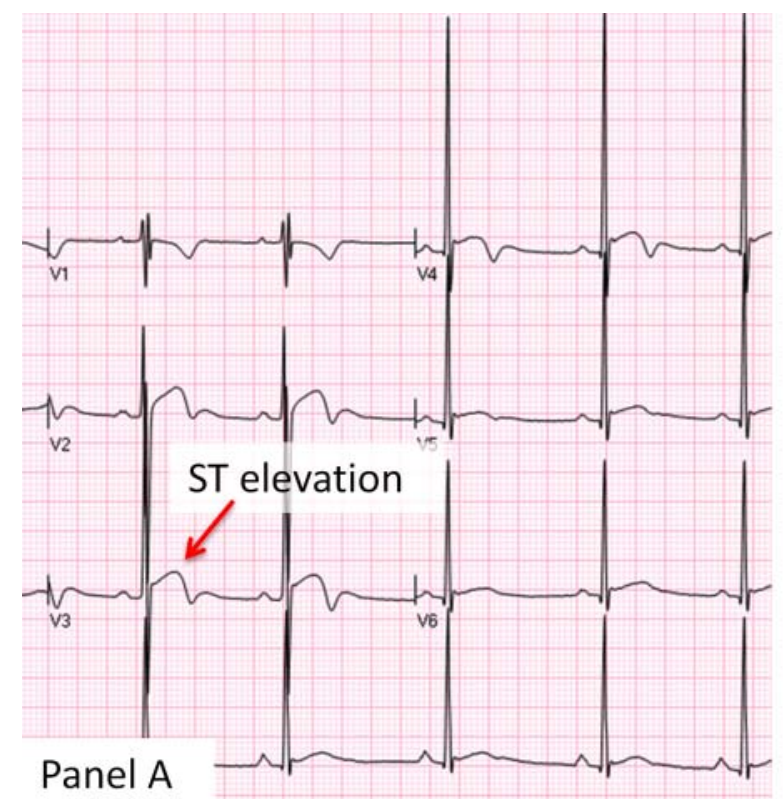

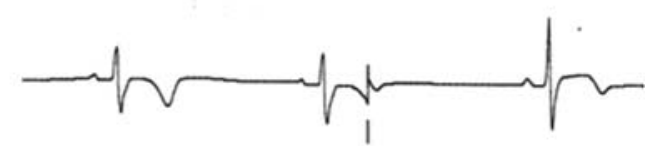

v2

vs

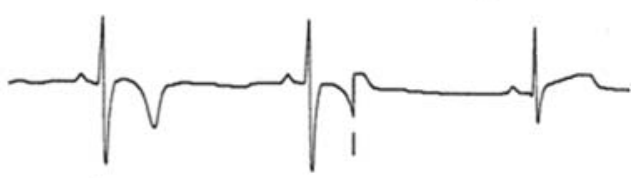

v3 Flat ST segment v6

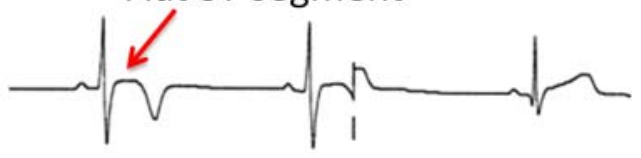

Panel B

Figure 13 (A) Normal variant repolarisation changes in a black/African athlete characterised by domed ST segment elevation and T wave inversion in V1-V4. (B) Pathological T wave inversion in V1-V3. Note the isoelectric ST segment. The absence of ST segment elevation prior to T wave inversion makes this ECG abnormal. Additional testing is required to rule out arrhythmogenic right ventricular cardiomyopathy. This figure is only reproduced in colour in the online version. 
cause of heart failure, the inability of the heart to meet the demands of the body. DCM can be caused by a variety of aetiologies ranging from coronary artery disease, infections/myocarditis, toxins (alcohol and other drugs), metabolic or endocrine abnormalities, autoimmune disorders, infiltrative processes and a variety of genetic/inheritable disorders. In many cases, a distinct cause of the compromised heart muscle is never found. DCM can be asymptomatic, or it can result in symptoms of exercise intolerance, shortness of breath, swelling, congestive heart failure or SCD from ventricular arrhythmia.

\section{Prevalence}

The exact prevalence of DCM in the general population is not known and estimates depend on the population demographics and the cut-off used to define LV dysfunction. ${ }^{55}$ The majority of cases with early stages of LV dysfunction may be asymptomatic. $^{56}$ DCM prevalence is increased with older age, male gender and the presence of cardiovascular risk factors. Among a relatively young, healthy population without coronary risk factors, asymptomatic LV dysfunction was present in about $0.2 \%$ of individuals. ${ }^{57}$

\section{Contribution as a cause of SCD}

DCM is associated with an increased risk of ventricular arrhythmias and sudden cardiac arrest, with highest risk seen among individuals with severe LV dysfunction. Notably, SCD can occur even in the absence of heart failure symptoms. In the Framingham Heart Study, the death rate from asymptomatic LV dysfunction was $6.5 \%$ per 100 person-years, with $53 \%$ of deaths occurring prior to the onset of symptoms, emphasising the importance of screening for this disorder. ${ }^{58}$ DCM accounts for about $2 \%$ of cases in a series of young athletes with sudden cardiac arrest. ${ }^{2}$

\section{Diagnostic criteria}

DCM is diagnosed on non-invasive imaging (echocardiography or cardiac MRI) by detecting LV chamber enlargement and decreased LV systolic (contractile) function. Systolic function is usually quantified using either fractional shortening (FS) or ejection fraction (EF), but there is no consensus cut-off definition of DCM based on these parameters. Normal ranges vary by lab and imaging modality.

\section{Abnormal ECG findings in DCM}

Several studies have examined ECG abnormalities in patients with symptomatic DCM, but few have reported findings among non-ischaemic DCM or among individuals with asymptomatic LV dysfunction. ${ }^{59-61}$ Overall, approximately $90 \%$ of individuals with DCM have abnormal ECG findings (figures 14 and 15). Patients with prior myocardial infarction may manifest pathological Q waves. Among individuals with non-ischaemic cardiomyopathy, the most common abnormalities seen include voltage criteria for LVH (33-40\%), TWIs (25-45\%), LAE (15-33\%), LAD (15-25\%), pathological Q waves (10-25\%), LBBB (9$25 \%)$, premature ventricular contractions (5-10\%) and RBBB (4\%). ${ }^{62}$ These findings are non-specific for DCM. Goldberger has proposed a triad of findings that may offer more specificity: (1) LVH in the anterior precordial leads; (2) low limb lead voltage and (3) poor precordial $\mathrm{R}$ wave progression. ${ }^{63}$ Given the overlap of some of these findings with physiological ECG changes found in athlete's heart, distinctly abnormal ECG criteria unrelated to regular training and requiring additional evaluation to rule out an underlying cardiomyopathy are listed in table 1.

\section{Evaluation of suspected DCM}

When DCM is suspected, further evaluation of LV size and function is required. Echocardiography provides assessment of cardiac structure and function, including FS and/or EF, and is the first test of choice under most circumstances. Contrast echocardiography or cardiac MRI should be considered when image resolution is reduced.

Athletes may have LV chamber enlargement as a part of physiological adaptation to exercise. ${ }^{64}$ This is most often seen in

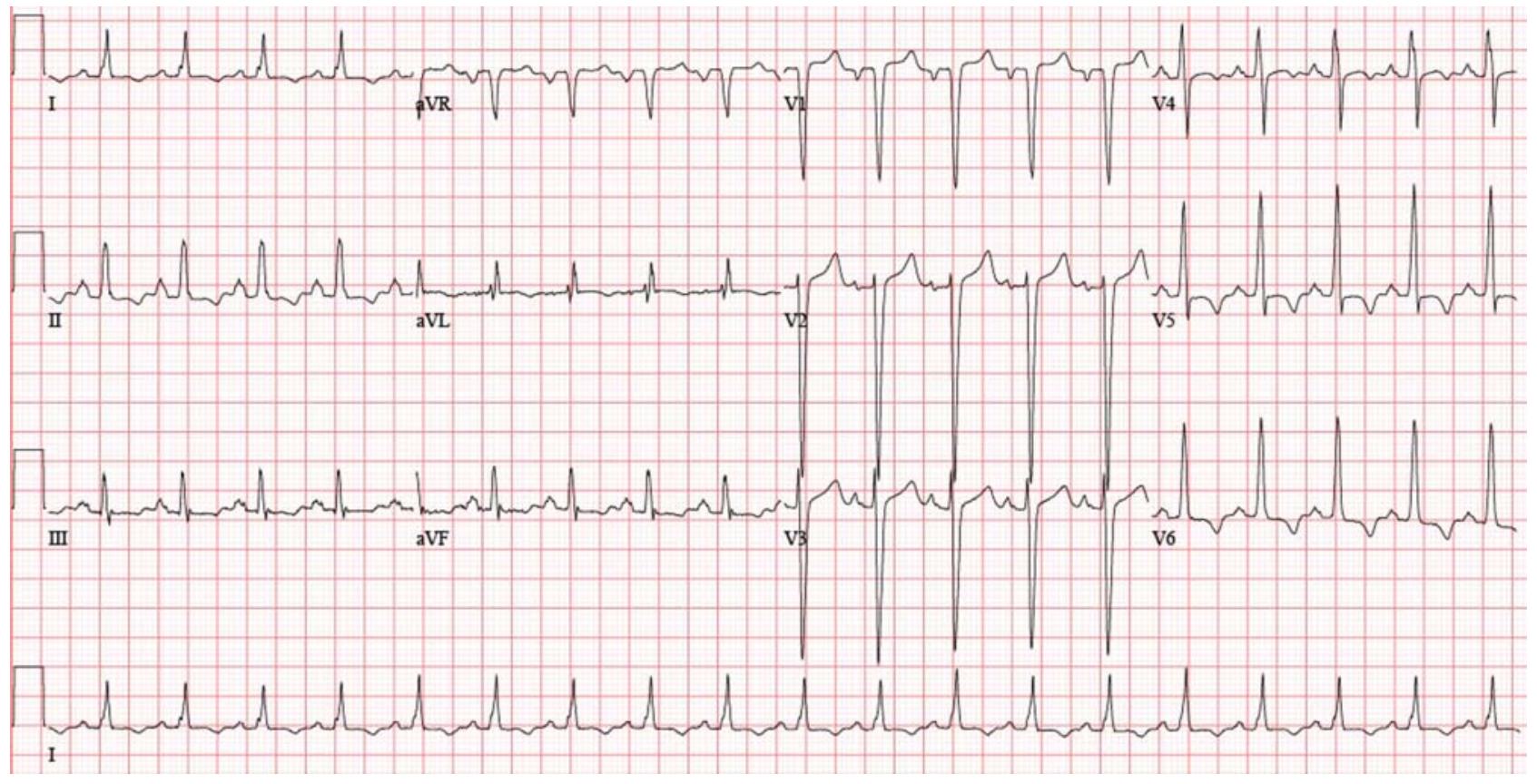

Figure 14 ECG from a patient with idiopathic dilated cardiomyopathy. Note the resting sinus tachycardia, left atrial enlargement, T wave inversions in the lateral limb (I and aVL) and precordial (V5-V6) leads and deep S waves in V1-V3 (part of Goldberger's triad). This figure is only reproduced in colour in the online version. 


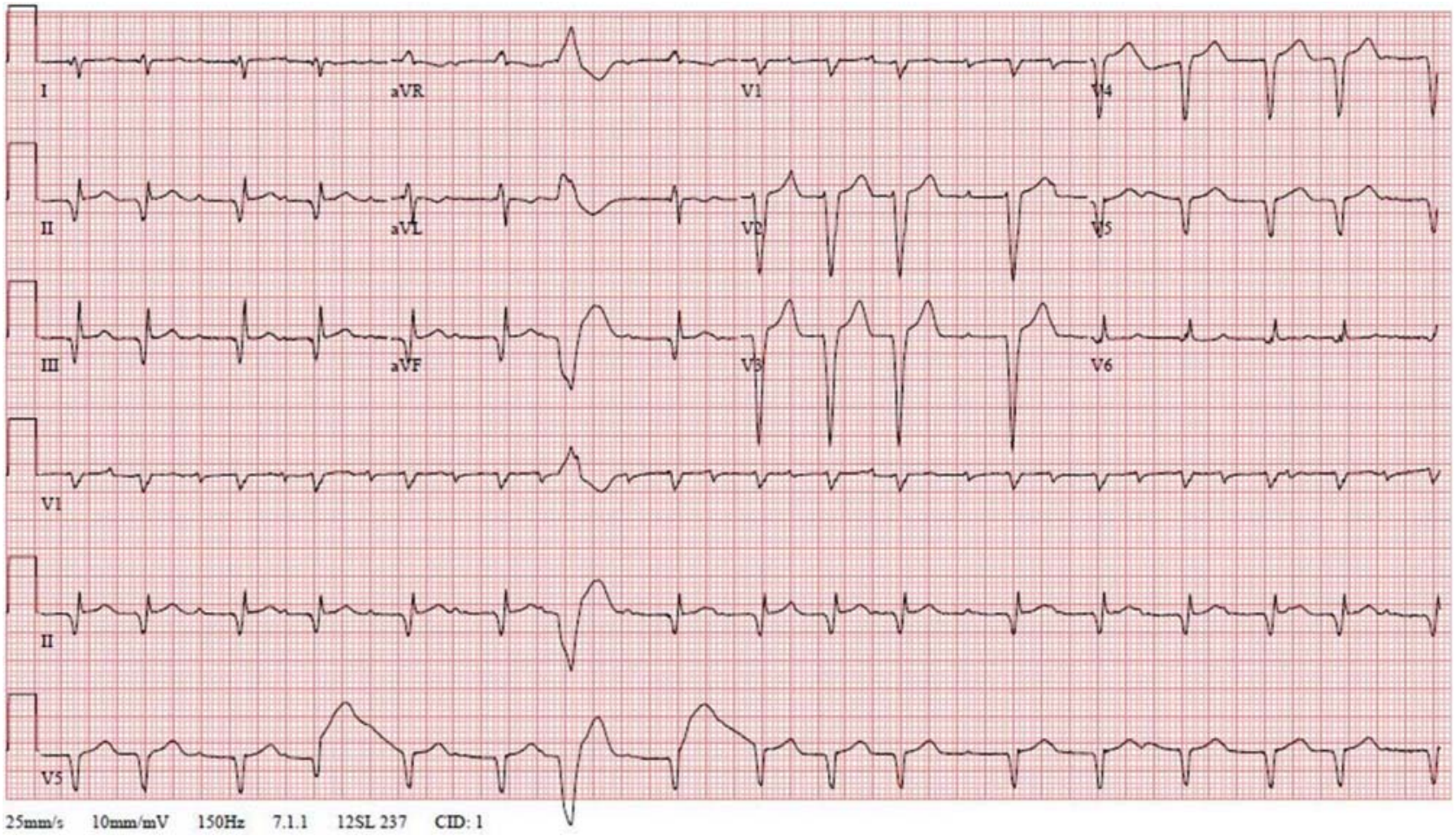

Figure 15 ECG of a young patient with dilated cardiomyopathy. Note inferior Q waves (II, III and aVF), poor R wave progression across the precordial leads with deep S waves in V1-V3, and a single premature ventricular complex. High-degree AV block is also present. This figure is only reproduced in colour in the online version.

athletes participating in endurance sports such as cycling, crosscountry skiing or rowing. Mild reduction in LV contractility (EF $40-50 \%)$ is seen in a minority of athletes with LV cavity dilation, but is not an invariable component of physiological adaptation to exercise. ${ }^{64}{ }^{65}$ Stress echocardiography can assess myocardial performance at submaximal or peak exercise which may help differentiate those with low normal or borderline systolic function, as systolic function is more likely to normalised in athlete's heart than in DCM. Thus, LV dilatation and measures of systolic function should be interpreted carefully and in the context of the athlete's level and amount of endurance training. All patients with DCM should be referred to a cardiologist for further aetiological evaluation, including assessment for myocardial ischaemia and infiltrative disorders.

\section{NON-COMPACTION}

LVNC is a heart muscle disorder in which loosely organised myocardial fibres fail to condense into a compact layer resulting in increased myocardial trabeculations and thinning of the compact myocardium (figure 16). ${ }^{66}{ }^{67} \mathrm{LVNC}$ can occur along with other congenital or embryological abnormalities, or can be found in isolation, and can be due to underlying gene mutations. However, the majority of LVNC remains genetically elusive. This disturbance of myocardial structure leads to progressive weakening of heart muscle contraction (lower EF) with ventricular dilation. Therefore, LVNC should be distinguished from DCM. Blood clots may also form within the trabecular recesses, increasing the risk for embolic strokes.

\section{Prevalence}

The exact prevalence of isolated LVNC is unknown, but is thought to be $<0.1-0.2 \%$. Reasons for the uncertainty in prevalence include challenges in imaging the non-compaction and disagreement regarding diagnostic criteria.

\section{Contribution as a cause of SCD}

LVNC is associated with an increased risk of abnormal heart rhythms and sudden cardiac arrest. ${ }^{68}$ LVNC is a rare $(<1 \%)$ cause of SCD in a series of young athletes. ${ }^{2}$

\section{Diagnostic criteria}

Several sets of diagnostic criteria for isolated LVNC exist, but remain controversial. ${ }^{69-73}$ These criteria are based generally on echocardiography or cardiac MRI findings of an increased ratio of trabeculations to compact myocardium. These criteria have

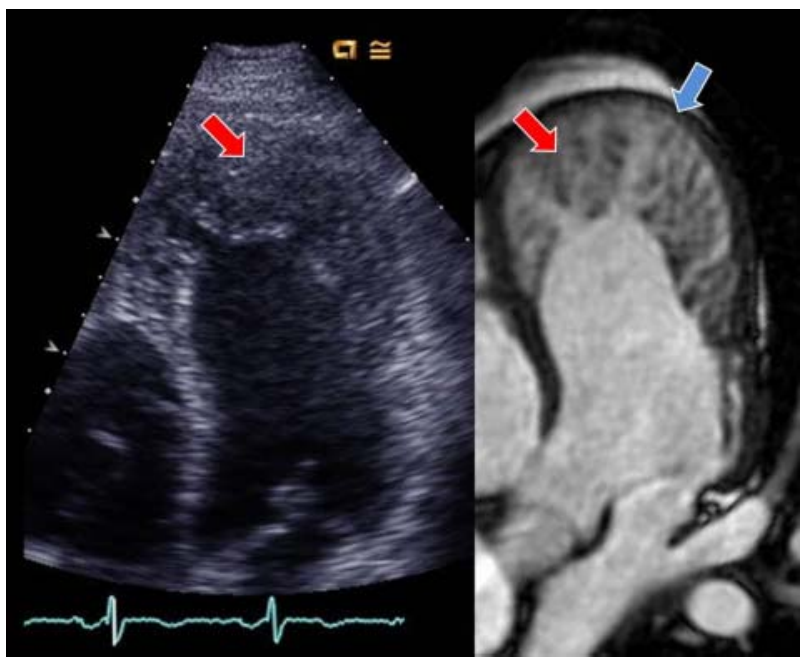

Figure 16 Echocardiographic (left panel) and cardiac MRI (right panel) of isolated left ventricular non-compaction. Note the prominent trabeculations in the left ventricular apex (red arrow), with a thin layer of compact myocardium (blue arrow) and prominent intertrabecular recesses. This figure is only reproduced in colour in the online version. 
been called into question recently for being non-specific, particularly among black individuals who have relatively greater degrees of myocardial trabeculations and among athletes. ${ }^{74}$

\section{Abnormal ECG findings in isolated LVNC}

ECG abnormalities in isolated LVNC are common but nonspecific (figures 17 and 18). In a series of 78 patients with a clinical diagnosis of LVNC, only $13 \%$ had a normal ECG. ${ }^{75}$ The most common abnormalities in this series included repolarisation changes (72\%), QT prolongation (52\%), ST segment depression (51\%), TWI (41\%), LVH voltage criteria (38\%), IVCD (31\%) including LBBB (19\%) and RBBB (3\%), and LAE $(26 \%) .{ }^{75}$ Given the overlap of some of these findings with physiological ECG changes found in athlete's heart, abnormal ECG criteria requiring additional evaluation to rule out an underlying cardiomyopathy are listed in table 1.

\section{Evaluation of suspected isolated LVNC}

Echocardiography is usually the first investigation in the evaluation of ECG abnormalities suggestive of cardiomyopathy. The diagnosis and evaluation of suspected LVNC is quite challenging, and therefore patients should be referred to a cardiovascular specialist familiar with LVNC. Cardiac MRI provides a more detailed and accurate assessment of myocardial trabeculations and is recommended in cases with concerning or borderline findings on echocardiography. A smaller absolute thickness of compacted myocardium and the presence of LV dysfunction favour the diagnosis of LVNC. In select cases, LV angiography may be used to help delineate hypertrabeculation from compacted myocardium. Holter monitoring or more extended ambulatory monitoring for arrhythmias also should be considered. In some cases, genetic testing may inform the diagnostic evaluation.

\section{OTHER ECG FINDINGS POSSIBLY SUGGESTIVE OF A CARDIOMYOPATHY}

Several additional ECG abnormalities including RBBB, nonspecific IVCD with QRS duration $<140 \mathrm{~ms}$, and isolated (one per tracing) ectopic/premature ventricular contractions (PVCs) have been associated with an underlying cardiomyopathy in non-athletic populations. ${ }^{76-83}$ However, these findings are also more common in trained athletes without an underlying heart disease than among the general population. ${ }^{26}{ }^{84-87}$ Each of these findings, particularly when observed in an asymptomatic athlete with no family history suggestive of heritable heart disease, has a low-positive predictive value for the cardiomyopathic conditions associated with an increased risk of SCD during exercise. As such, none of these ECG patterns, when found in isolation in asymptomatic athletes, clearly necessitate further evaluation. However, in athletes with cardiovascularrelated symptoms or a family history of sudden death or suspected cardiomyopathy, each of these findings should prompt additional evaluation to evaluate for cardiomyopathy.

\section{Right bundle branch block}

RBBB is defined as a QRS complex $\geq 120 \mathrm{~ms}$ in association with a terminal (final component of the QRS complex) $\mathrm{R}^{\prime}$ wave in lead V1 and terminal S waves in leads I, aVL and V6 (figure 19). The $\mathrm{R}^{\prime}$ may extend into lead V2 but is typically absent in other precordial leads. T waves in typical RBBB are in the same direction as the terminal QRS forces and are thus inverted in leads with an $\mathrm{R}^{\prime}(\mathrm{V} 1 \pm \mathrm{V} 2)$. A QRS complex duration of

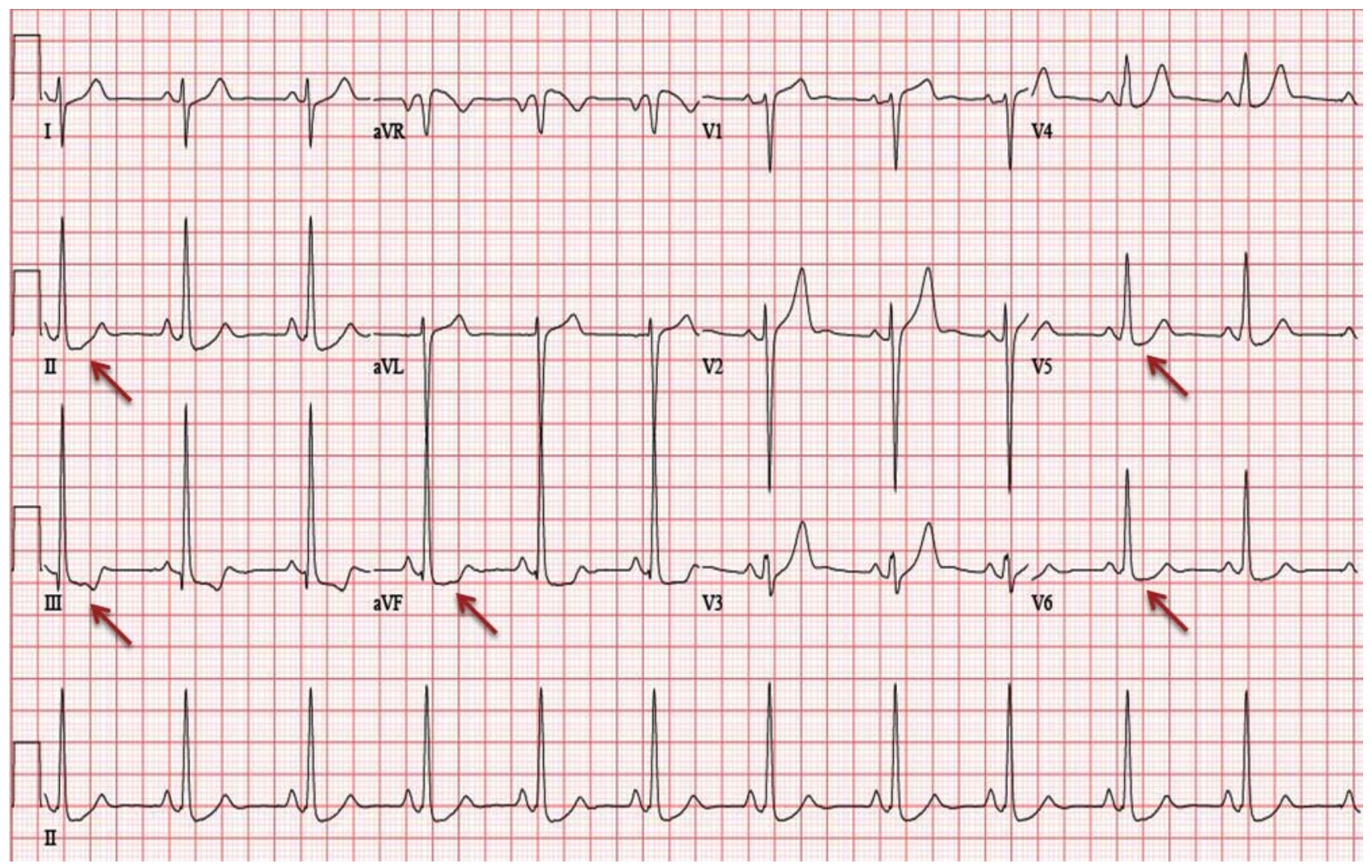

Figure 17 ECG from a patient with isolated left ventricular non-compaction. Note the ST segment depression in the inferolateral leads (II, III, aVF and V5-V6). This figure is only reproduced in colour in the online version. 


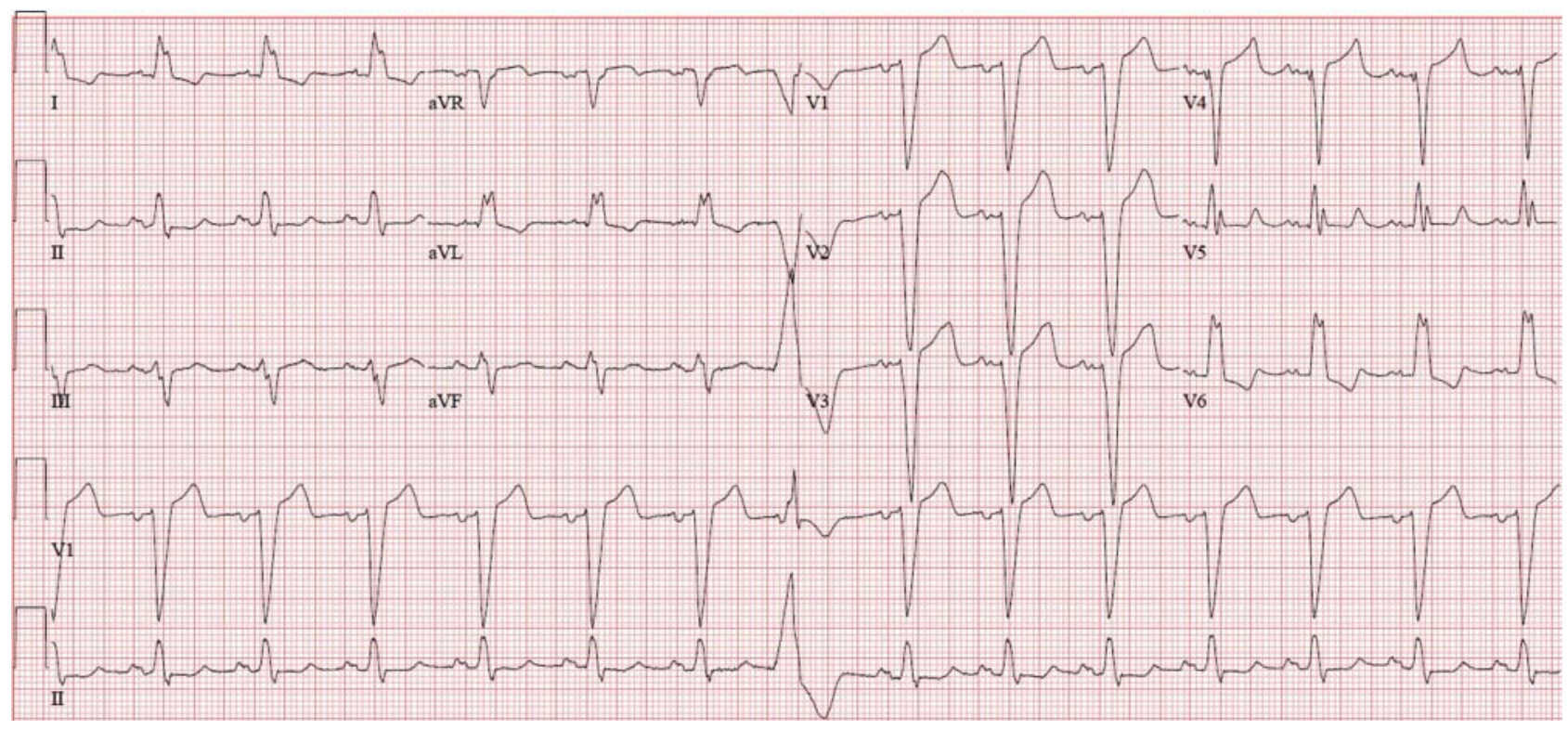

Figure 18 ECG from a patient with isolated left ventricular non-compaction, demonstrating left atrial enlargement with a left bundle branch block. An isolated premature ventricular complex is also present. These findings warrant further testing to evaluate for cardiomyopathy. This figure is only reproduced in colour in the online version.

$100-119$ ms with these morphological features is termed an incomplete RBBB.

Although RBBB may be present in various forms of heart disease, complete and incomplete RBBB are found commonly among trained athletes without underlying heart disease. This ECG pattern has been shown to reflect the exercise-induced right ventricular remodelling common in endurance sport athletes. ${ }^{88}$ In asymptomatic athletes with an isolated complete or incomplete RBBB, no further diagnostic evaluation is required. In contrast, athletes presenting with symptoms suggestive of cardiomyopathy, a family history of sudden death or suspected cardiomyopathy, an RBBB with atypical features (extensive TWIs, ST-segment elevation or a markedly prolonged $\mathrm{R}^{\prime}$ ), or a $\mathrm{RBBB}$ in conjunction with other abnormal ECG findings should be further evaluated.

\section{Non-Specific intra-ventricular conduction delay}

IVCD is defined as a QRS complex $>110 \mathrm{~ms}$ that does not have morphological features consistent with either LBBB or RBBB. IVCD has been documented among patients with

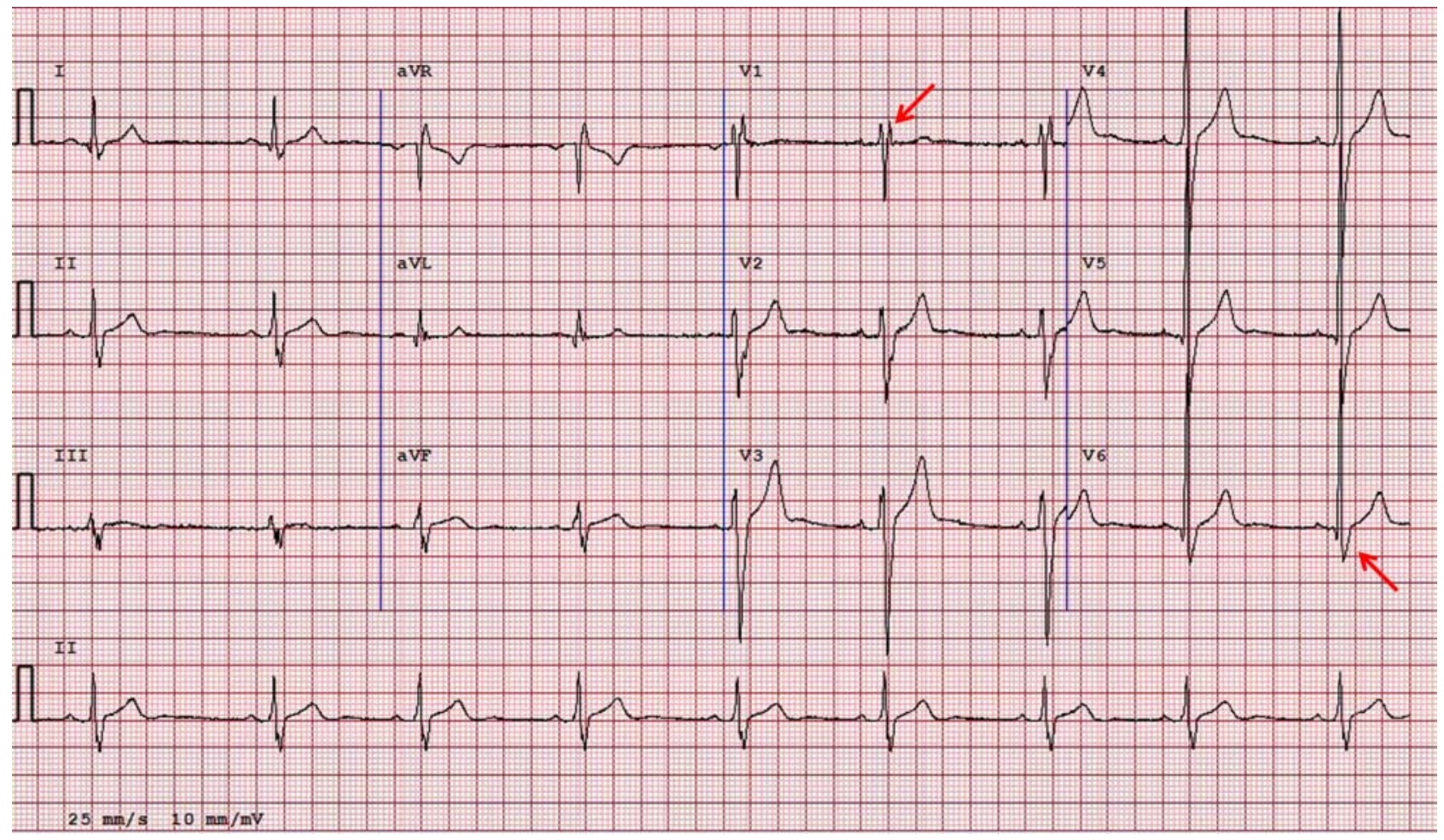

Figure 19 ECG showing complete right bundle branch block with an $\mathrm{R}^{\prime}$ wave in V1, terminal $\mathrm{S}$ wave in V6, and a QRS duration of 128 ms. This figure is only reproduced in colour in the online version. 
cardiomyopathy, but is also frequently seen in healthy athletes. The physiology underlying IVCD in athletes remains incompletely understood but likely includes some combination of neurally mediated conduction fibre slowing and increased myocardial mass.

Digital analysis of QRS duration can outperform standard visual measurement because the first onset and last offset in all of the leads can be considered. ${ }^{6}$ In asymptomatic athletes with an isolated IVCD with a QRS duration of 100-139 ms, no further diagnostic evaluation is required. In contrast, athletes presenting with symptoms suggestive of cardiomyopathy, a family history of sudden death or suspected cardiomyopathy, an IVCD with marked QRS prolongation ( $\geq 140 \mathrm{~ms}$ ) or an IVCD in tandem with other abnormal ECG findings should be further evaluated.

\section{Isolated premature ventricular contractions}

PVCs are electrical impulses that originate from myocardial tissue below the AV node. They are defined as QRS complexes $>100 \mathrm{~ms}$ that are not preceded by a triggering $\mathrm{p}$-wave. PVCs may reflect pathological myocardial 'irritability' due to a cardiomyopathy, an underlying systemic disease process, or may be a completely benign normal variant. PVCs are common in athletes with high vagal tone and resting bradycardia and may increase in frequency in parallel with physical fitness. A single PVC captured during a routine 12-lead ECG in an asymptomatic athlete does not require further evaluation, unless the athlete performs a high-intensity endurance sport (mainly cycling, triathlon, rowing or swimming). In this select group of high-intensity endurance athletes, a single PVC, especially if it has an LBBB morphology, may be a hallmark of 'exercise-induced' ARVC, and further evaluation should be considered. ${ }^{46-48}$ The presence of PVCs in an athlete with cardiovascular symptoms or a family history of sudden death or suspected cardiomyopathy should prompt further evaluation. In addition, multiple PVCs (2 or more) during a single ECG tracing (10 s), multifocal PVCs or PVCs found in tandem with other abnormal ECG findings should be further evaluated.

\section{PULMONARY HYPERTENSION}

Pulmonary hypertension (PHT) is caused by a variety of aetiologies that result in elevation in the pulmonary artery pressure (mean pulmonary artery pressure greater than or equal to $25 \mathrm{~mm} \mathrm{Hg}$ ) and elevation in the pulmonary vascular resistance. ${ }^{89}$ As a result of the increased afterload on the right heart, patients are predisposed to develop right heart failure and are at risk for sudden death. PHT is a rare cause of sudden death in athletes but may be suggested by ECG abnormalities and thus a clinically relevant finding in the cardiovascular care of athletes.

\section{ECG findings in pulmonary hypertension}

The ECG findings in PHT are due to physiological and anatomic adaptions of the right heart as a result of elevated pulmonary artery pressures and/or pulmonary vascular resistance. Findings suggestive of PHT include right ventricular hypertrophy (RVH), right axis deviation, right ventricular strain and right atrial enlargement (figure 20). ${ }^{89-91}$ In adults with idiopathic PHT, $87 \%$ demonstrated $\mathrm{RVH}$ and $79 \%$ demonstrated right axis deviation. ${ }^{89}$ However, in patients with PHT, the ECG remains an inadequate screening tool to completely rule out the presence of this disease. ${ }^{92}$

\section{Right ventricular hypertrophy pattern}

RVH pattern is defined as an R wave in lead V1 plus S wave in V5 greater than $1.05 \mathrm{mV}(10.5 \mathrm{~mm}$ at standard amplification) AND right axis deviation $>120^{\circ}$ (figure 20). Additional criteria for RVH associated with PHT include a tall R wave and small S wave with $\mathrm{R} / \mathrm{S}$ ratio greater than 1 in lead $\mathrm{V} 1$ and a $\mathrm{qR}$ complex in lead V1. The presence of RHV pattern on ECG should prompt further investigation in the athlete.

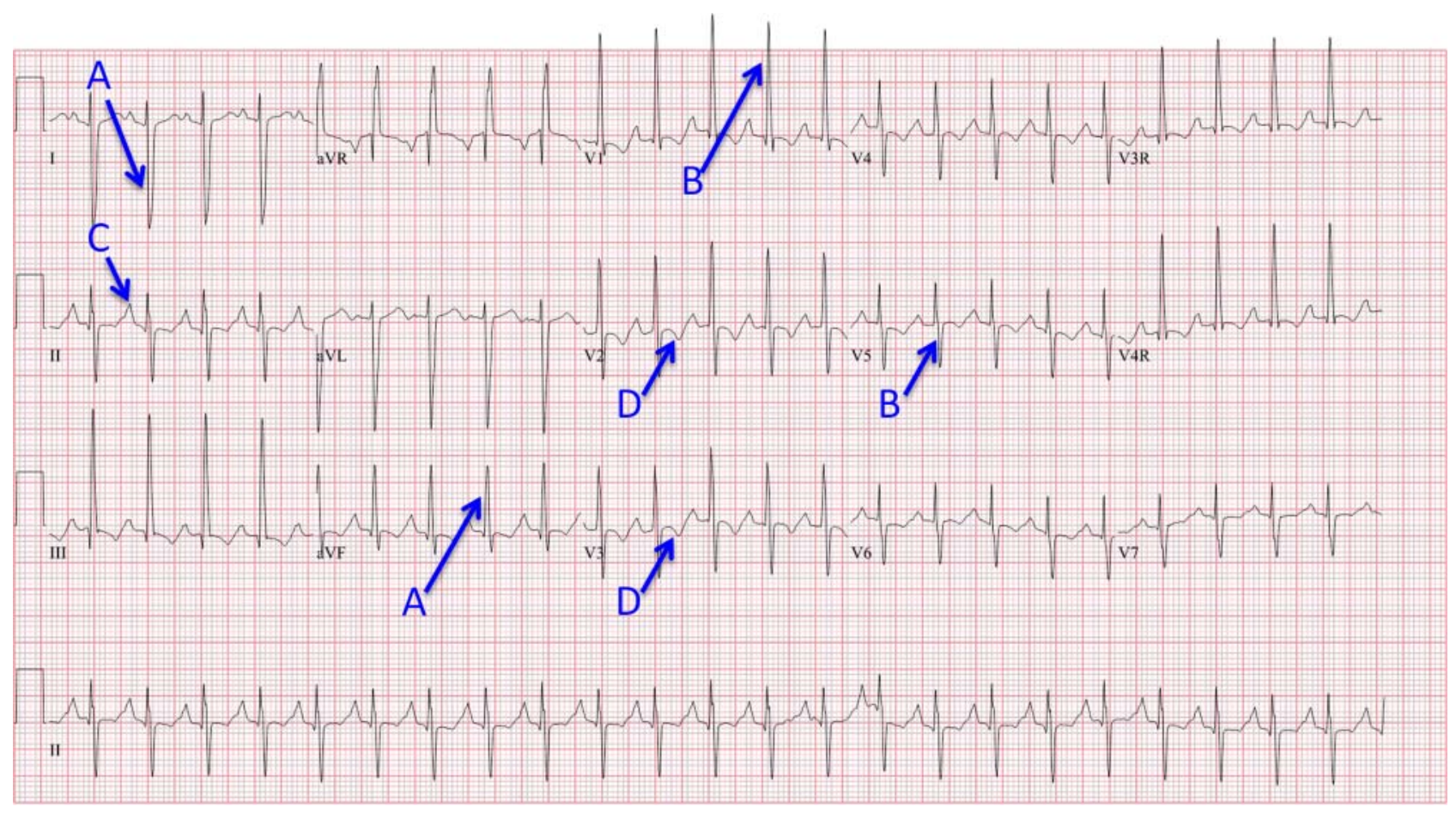

Figure 20 ECG from a patient with pulmonary hypertension demonstrating evidence of right-axis deviation $>120^{\circ}(A)$, right ventricular hypertrophy (B), right atrial enlargement (C) and right ventricular strain (D). This figure is only reproduced in colour in the online version. 
Right axis deviation

Right-axis deviation is defined as a frontal plane QRS axis of $>120^{\circ}$ (figure 20 ).

Right atrial enlargement

Right atrial enlargement is defined as a $\mathrm{P}$ wave greater than or equal to $2.5 \mathrm{~mm}$ in leads II, III and aVF (figure 20).

\section{RV strain}

Right ventricular 'strain' is defined as ST depression and TWI in the right precordial leads (V1-V3) (figure 20). As with LVH, these ST-T changes are referred to as 'secondary ST-T abnormalities.'

\section{Evaluation of suspected pulmonary hypertension}

Evaluation should include clinical assessment with appropriate diagnostic testing. Pulmonary artery pressures often can be assessed by Doppler echocardiography, and both echocardiography and cardiac MRI can evaluate RVH and function, and assess for secondary causes of PHT such as intracardiac shunts. Definitive diagnosis of PHT is made by cardiac catheterisation.

\section{CONCLUSIONS}

The cardiomyopathies are a heterogeneous group of heart muscle diseases associated with important clinical implications. In aggregate, HCM, ARVC, DCM and LVNC underlie the majority of autopsy-positive sudden death cases in young athletes. Each of these cardiomyopathies can manifest in athletes with a broad spectrum of clinical severity ranging from completely asymptomatic to markedly symptomatic disease with associated exercise limitations. The ECG plays an important role in the cardiovascular assessment of athletes given its capacity to detect cardiomyopathies. As delineated in this paper, there is a concise list of ECG findings that may indicate the presence of an underlying cardiomyopathic condition. Importantly, these ECG findings are not characteristic of the benign exercise-induced cardiac remodelling common in athletes, and, thus, the ECG can be useful for differentiating physiological cardiac enlargement in athletes from pathological myocardial disease.

Clinicians charged with the cardiovascular care of athletes should be familiar with the ECG findings associated with cardiomyopathy. During pre-participation screening that includes the use of ECG, asymptomatic athletes with any of these abnormal findings should undergo further testing. Athletes presenting with symptoms that may be indicative of an underlying cardiomyopathy (ie, exercise intolerance, inappropriate exertional dyspnoea, chest pain, palpitations or syncope) should undergo a prompt evaluation including an ECG. The symptomatic athlete with an ECG suggestive of a cardiomyopathy requires a comprehensive and definitive assessment that will include some combination of non-invasive cardiac imaging, exercise testing and ambulatory rhythm monitoring. This evaluation should be conducted by a sports medicine team that includes a cardiovascular specialist familiar with cardiomyopathic diseases and with experience in caring for athletes.

\section{Additional resources}

For a free online training module on ECG interpretation in athletes, please visit: http://learning.bmj.com/ECGathlete

For the November 2012 BJSM supplement on 'Advances in Sports Cardiology,' please visit: http://bjsm.bmj.com/content/46/ Suppl_1.toc

\section{Author affiliations}

${ }^{1}$ Department of Family Medicine, University of Washington, Seattle, Washington, USA

${ }^{2}$ Division of Cardiovascular Medicine, Stanford University School of Medicine, Palo Alto, California, USA

${ }^{3}$ Division of Cardiology, Massachusetts General Hospital, Boston, Massachusetts, USA

${ }^{4}$ Department of Cardiology, Swedish School of Sports and Health Sciences, Karolinska University Hospital, Stockholm, Sweden

${ }^{5}$ Department of Cardiac, Thoracic, and Vascular Sciences, University of Padua, Padova, Italy

${ }^{6}$ Division of Cardiology, University of Washington, Seattle, Washington, USA

${ }^{7}$ Division of Cardiology, The Children's Hospital of Philadelphia, Philadelphia, Pennsylvania, USA

${ }^{8}$ Department of Medicine, Institute of Sport Medicine and Science, Rome, Italy

${ }^{9}$ Departments of Medicine, Pediatrics, and Molecular Pharmacology and

Experimental Therapeutics, Mayo Clinic, Rochester, Minnesota, USA

${ }^{10}$ Divisions of Cardiovascular Diseases and Pediatric Cardiology, Mayo Clinic,

Rochester, Minnesota, USA

${ }^{11}$ Department of Athletics, University of Connecticut, Storrs, Connecticut, USA

${ }^{12}$ Department of Family Medicine, Eisenhower Army Medical Center, Fort Gordon,

Georgia, USA

${ }^{13}$ Division of Cardiovascular Diseases, Mayo Clinic, Rochester, Minnesota, USA

${ }^{14}$ Division of Sports Medicine, University of California Los Angeles, Los Angeles,

California, USA

${ }^{15}$ Department of Pediatrics, Emory University School of Medicine, Children's Healthcare of Atlanta, Atlanta, Georgia, USA

${ }^{16}$ Department of Cardiovascular Sciences, University of Leuven, Leuven, Belgium

${ }^{17}$ Midwest Heart Foundation, Oakbrook Terrace, Illinois, USA

${ }^{18}$ Department of Family and Community Medicine, University of Arizona, Tucson Arizona, USA

${ }^{19}$ Division of Cardiology, Seattle Children's Hospital, Seattle, Washington, USA

${ }^{20}$ Division of Cardiology, University Hospital Zurich, Zurich, Switzerland

${ }^{21}$ Department of Cardivascular Sciences, St. George's University of London, London, UK

${ }^{22}$ Cardiology Department, Hospital de Clínicas de Porto Alegre, Porto Alegre, Brazil

${ }^{23}$ Department of Sports Medicine, ASPETAR, Qatar Orthopedic and Sports Medicine Hospital, Doha, Qatar

Competing interests None.

Funding None.

Provenance and peer review Commissioned; internally peer reviewed.

\section{REFERENCES}

1 Maron BJ, Doerer JJ, Haas TS, et al. Sudden deaths in young competitive athletes: analysis of 1866 deaths in the United States, 1980-2006. Circulation 2009;119:1085-92.

2 Maron BJ. Sudden death in young athletes. N Engl J Med 2003;349:1064-75.

3 Corrado D, Basso C, Schiavon M, et al. Screening for hypertrophic cardiomyopathy in young athletes. N Engl J Med 1998;339:364-9.

4 Corrado D, Biffi A, Basso C, et al. 12-Lead ECG in the athlete: physiological versus pathological abnormalities. Br J Sports Med 2009;43:669-76.

5 Corrado D, Pelliccia A, Heidbuchel $H$, et al. Recommendations for interpretation of 12-lead electrocardiogram in the athlete. Eur Heart J 2010;31:243-59.

6 Uberoi A, Stein R, Perez MV, et al. Interpretation of the electrocardiogram of young athletes. Circulation 2011;124:746-57.

7 Williams ES, Owens DS, Drezner JA, et al. Electrocardiogram interpretation in the athlete. Herzschrittmacherther Elektrophysiol 2012;23:65-71.

8 Drezner J. Standardised criteria for ECG interpretation in athletes: a practical tool. Br J Sports Med 2012;46(Suppl I): i6-8.

9 Marek J, Bufalino V, Davis J, et al. Feasibility and findings of large-scale electrocardiographic screening in young adults: data from 32561 subjects. Heart Rhythm 2011;8:1555-9.

10 Drezner JA, Ackerman MJ, Anderson J, et al. Electrocardiographic interpretation in athletes: the 'seattle criteria'. Br J Sports Med 2013;47.

11 Drezner JA, Fischbach $\mathrm{P}$, Foelicher $\mathrm{V}$, et al. Normal electrocardiographic findings: recognizing physiologic adaptations in athletes. Br J Sports Med 2013;47.

12 Maron BJ. Hypertrophic cardiomyopathy. Lancet 1997;350:127-33.

13 Maron BJ, Shirani J, Poliac LC, et al. Sudden death in young competitive athletes. Clinical, demographic, and pathological profiles. JAMA 1996;276:199-204.

14 Basavarajaiah S, Wilson M, Whyte $G$, et al. Prevalence of hypertrophic cardiomyopathy in highly trained athletes: relevance to pre-participation screening. J Am Coll Cardiol 2008;51:1033-9.

15 de Noronha SV, Sharma S, Papadakis M, et al. Aetiology of sudden cardiac death in athletes in the United Kingdom: a pathological study. Heart 2009;95:1409-14. 
16 Eckart RE, Shry EA, Burke AP, et al. Sudden death in young adults an autopsy-based series of a population undergoing active surveillance. J Am Coll Cardiol 2011;58:1254-61.

17 Meyer L, Stubbs B, Fahrenbruch C, et al. Incidence, causes, and survival trends from cardiovascular-related sudden cardiac arrest in children and young adults 0 to 35 years of age: a 30-year review. Circulation 2012;126:1363-72.

18 Maron BJ. Distinguishing hypertrophic cardiomyopathy from athlete's heart physiological remodelling: clinical significance, diagnostic strategies and implications for preparticipation screening. Br J Sports Med 2009;43:649-56.

19 Ryan MP, Cleland JG, French JA, et al. The standard electrocardiogram as a screening test for hypertrophic cardiomyopathy. Am J Cardiol 1995;76:689-94.

20 Maron BJ, Roberts WC, Epstein SE. Sudden death in hypertrophic cardiomyopathy: a profile of 78 patients. Circulation 1982;65:1388-94.

21 Rowin EJ, Maron BJ, Appelbaum E, et al. Significance of false negative electrocardiograms in preparticipation screening of athletes for hypertrophic cardiomyopathy. Am J Cardiol 2012;110:1027-32.

22 Lakdawala NK, Thune JJ, Maron BJ, et al. Electrocardiographic features of sarcomere mutation carriers with and without clinically overt hypertrophic cardiomyopathy. Am J Cardiol 2011;108:1606-13.

23 Papadakis M, Carre F, Kervio G, et al. The prevalence, distribution, and clinical outcomes of electrocardiographic repolarization patterns in male athletes of African/ Afro-Caribbean origin. Eur Heart J 2011;32:2304-13.

24 Papadakis M, Basavarajaiah S, Rawlins J, et al. Prevalence and significance of T-wave inversions in predominantly Caucasian adolescent athletes. Eur Heart J 2009:30:1728-35.

25 Le W, Wheeler MT, Mandic S, et al. Addition of the electrocardiogram to the preparticipation examination of college athletes. Clin J Sport Med 2010;20: 98-105.

26 Pelliccia A, Maron BJ, Culasso F, et al. Clinical significance of abnormal electrocardiographic patterns in trained athletes. Circulation 2000;102:278-84.

27 Di Paolo FM, Schmied C, Zerguini YA, et al. The athlete's heart in adolescent Africans: an electrocardiographic and echocardiographic study. J Am Coll Cardiol 2012;59:1029-36.

28 Migliore F, Zorzi A, Michieli P, et al. Prevalence of cardiomyopathy in Italian asymptomatic children with electrocardiographic T-wave inversion at preparticipation screening. Circulation 2012;125:529-38.

29 Konno T, Shimizu M, Ino H, et al. Diagnostic value of abnormal Q waves for identification of preclinical carriers of hypertrophic cardiomyopathy based on a molecular genetic diagnosis. Eur Heart J 2004;25:246-51.

30 Pelliccia A, Culasso F, Di Paolo FM, et al. Prevalence of abnormal electrocardiograms in a large, unselected population undergoing pre-participation cardiovascular screening. Eur Heart J 2007;28:2006-10.

31 Sohaib SM, Payne JR, Shukla R, et al. Electrocardiographic (ECG) criteria for determining left ventricular mass in young healthy men; data from the LARGE Heart study. J Cardiovasc Magn Reson 2009:11:2.

32 Sathanandam S, Zimmerman F, Davis J, et al. Abstract 2484: ECG screening criteria for LVH does not correlate with diagnosis of hypertrophic cardiomyopathy. Circulation 2009;120:\$647

33 Weiner RB, Hutter AM, Wang F, et al. Performance of the 2010 European Society of Cardiology criteria for ECG interpretation in the athlete. Heart 2011;97:1573-7.

34 Gersh BJ, Maron BJ, Bonow RO, et al. ACCF/AHA guideline for the diagnosis and treatment of hypertrophic cardiomyopathy: a report of the American College of Cardiology Foundation/American Heart Association Task Force on Practice Guidelines. Circulation 2011;124:e783-831.

35 Maron MS, Maron BJ, Harrigan C, et al. Hypertrophic cardiomyopathy phenotype revisited after 50 years with cardiovascular magnetic resonance. J Am Coll Cardiol 2009;54:220-8.

36 Pelliccia A, Maron MS, Maron BJ. Assessment of left ventricular hypertrophy in a trained athlete: differential diagnosis of physiologic athlete's heart from pathologic hypertrophy. Prog Cardiovasc Dis 2012;54:387-96.

37 Rawlins J, Bhan A, Sharma S. Left ventricular hypertrophy in athletes. Eur J Echocardiogr 2009;10:350-6.

38 Maron BJ. Distinguishing hypertrophic cardiomyopathy from athlete's heart: a clinical problem of increasing magnitude and significance. Heart 2005;91:1380-2.

39 Maron BJ, Wolfson JK, Ciro E, et al. Relation of electrocardiographic abnormalities and patterns of left ventricular hypertrophy identified by 2-dimensional echocardiography in patients with hypertrophic cardiomyopathy. Am J Cardiol 1983;51:189-94

40 Thiene G, Nava A, Corrado D, et al. Right ventricular cardiomyopathy and sudden death in young people. N Engl J Med 1988;318:129-33.

41 Maron BJ, Niimura $H$, Casey SA, et al. Development of left ventricular hypertrophy in adults in hypertrophic cardiomyopathy caused by cardiac myosin-binding protein C gene mutations. J Am Coll Cardiol 2001;38:315-21.

42 Maron BJ, Seidman JG, Seidman CE. Proposal for contemporary screening strategies in families with hypertrophic cardiomyopathy. J Am Coll Cardiol 2004;44:2125-32.

43 Pelliccia A, Di Paolo FM, Quattrini FM, et al. Outcomes in athletes with marked ECG repolarization abnormalities. N Engl J Med 2008;358:152-61.
44 Basso C, Corrado D, Marcus Fl, et al. Arrhythmogenic right ventricular cardiomyopathy. Lancet 2009;373:1289-300

45 Corrado D, Basso C, Thiene G. Arrhythmogenic right ventricular cardiomyopathy: an update. Heart 2009;95:766-73.

46 Heidbuchel $H$, Hoogsteen J, Fagard R, et al. High prevalence of right ventricular involvement in endurance athletes with ventricular arrhythmias. Role of an electrophysiologic study in risk stratification. Eur Heart J 2003;24:1473-80.

47 La Gerche A, Robberecht C, Kuiperi C, et al. Lower than expected desmosomal gene mutation prevalence in endurance athletes with complex ventricular arrhythmias of right ventricular origin. Heart 2010;96:1268-74.

48 Heidbuchel $H$, Prior DL, Gerche AL. Ventricular arrhythmias associated with long-term endurance sports: what is the evidence? Br J Sports Med 2012:46(Suppl 1):i44-50.

49 Marcus FI, McKenna WJ, Sherrill D, et al. Diagnosis of arrhythmogenic right ventricular cardiomyopathy/dysplasia: proposed modification of the task force criteria. Circulation 2010;121:1533-41.

50 Nasir K, Bomma C, Tandri H, et al. Electrocardiographic features of arrhythmogenic right ventricular dysplasia/cardiomyopathy according to disease severity: a need to broaden diagnostic criteria. Circulation 2004;110:1527-34.

51 Steriotis AK, Bauce B, Daliento L, et al. Electrocardiographic pattern in arrhythmogenic right ventricular cardiomyopathy. Am J Cardiol 2009;103:1302-8.

52 Protonotarios N, Anastasakis A, Antoniades L, et al. Arrhythmogenic right ventricular cardiomyopathy/dysplasia on the basis of the revised diagnostic criteria in affected families with desmosomal mutations. Eur Heart J 2011;32:1097-104.

53 Kamath GS, Zareba W, Delaney J, et al. Value of the signal-averaged electrocardiogram in arrhythmogenic right ventricular cardiomyopathy/dysplasia. Heart Rhythm 2011;8:256-62.

54 Marcus Fl, Zareba W, Calkins H, et al. Arrhythmogenic right ventricular cardiomyopathy/dysplasia clinical presentation and diagnostic evaluation: results from the North American Multidisciplinary Study. Heart Rhythm 2009;6:984-92

55 Atherton JJ. Screening for left ventricular systolic dysfunction: is imaging a solution? JACC Cardiovasc Imaging 2010;3:421-8.

56 Ammar KA, Jacobsen SJ, Mahoney DW, et al. Prevalence and prognostic significance of heart failure stages: application of the American College of Cardiology/American Heart Association heart failure staging criteria in the community. Circulation 2007;115:1563-70.

57 Galasko Gl, Barnes SC, Collinson P, et al. What is the most cost-effective strategy to screen for left ventricular systolic dysfunction: natriuretic peptides, the electrocardiogram, hand-held echocardiography, traditional echocardiography, or their combination? Eur Heart J 2006:27:193-200.

58 Wang TJ, Evans JC, Benjamin EJ, et al. Natural history of asymptomatic left ventricular systolic dysfunction in the community. Circulation 2003;108:977-82.

59 Baig MK, Goldman JH, Caforio AL, et al. Familial dilated cardiomyopathy: cardiac abnormalities are common in asymptomatic relatives and may represent early disease. J Am Coll Cardiol 1998;31:195-201.

60 Grunig E, Tasman JA, Kucherer $\mathrm{H}$, et al. Frequency and phenotypes of familial dilated cardiomyopathy. J Am Coll Cardiol 1998;31:186-94.

61 Mahon NG, Murphy RT, MacRae CA, et al. Echocardiographic evaluation in asymptomatic relatives of patients with dilated cardiomyopathy reveals preclinical disease. Ann Intern Med 2005;143:108-15.

62 Surwicz B, Knilan T, eds. Chou's electrocardiography in clinical practice. Philadelphia: WB Saunders Company, 2001:256-8.

63 Goldberger AL. A specific ECG triad associated with congestive heart failure. Pacing Clin Electrophysiol 1982;5:593-9.

64 Pelliccia A, Culasso F, Di Paolo FM, et al. Physiologic left ventricular cavity dilatation in elite athletes. Ann Intern Med 1999;130:23-31.

65 Abergel E, Chatellier G, Hagege AA, et al. Serial left ventricular adaptations in world-class professional cyclists: implications for disease screening and follow-up. J Am Coll Cardiol 2004:44:144-9.

66 Paterick TE, Umland MM, Jan MF, et al. Left ventricular noncompaction: a 25-year odyssey. J Am Soc Echocardiogr 2012;25:363-75

67 Sarma RJ, Chana A, Elkayam U. Left ventricular noncompaction. Prog Cardiovasc Dis 2010;52:264-73.

68 Steffel J, Duru F. Rhythm disorders in isolated left ventricular noncompaction. Ann Med 2012;44:101-8.

69 Chin TK, Perloff JK, Williams RG, et al. Isolated noncompaction of left ventricular myocardium. A study of eight cases. Circulation 1990;82:507-13.

70 Jenni R, Oechslin E, Schneider J, et al. Echocardiographic and pathoanatomical characteristics of isolated left ventricular non-compaction: a step towards classification as a distinct cardiomyopathy. Heart 2001;86:666-71.

71 Jacquier $A$, Thuny $F$, Jop $B$, et al. Measurement of trabeculated left ventricular mass using cardiac magnetic resonance imaging in the diagnosis of left ventricular non-compaction. Eur Heart J 2010;31:1098-104.

72 Petersen SE, Selvanayagam JB, Wiesmann F, et al. Left ventricular non-compaction: insights from cardiovascular magnetic resonance imaging. J Am Coll Cardiol 2005; $46: 101-5$ 
73 Stollberger C, Kopsa W, Tscherney R, et al. Diagnosing left ventricular noncompaction by echocardiography and cardiac magnetic resonance imaging and its dependency on neuromuscular disorders. Clin Cardiol 2008;31:383-7.

74 Kohli SK, Pantazis AA, Shah JS, et al. Diagnosis of left-ventricular non-compaction in patients with left-ventricular systolic dysfunction: time for a reappraisal of diagnostic criteria? Eur Heart J 2008;29:89-95.

75 Steffel J, Kobza R, Oechslin E, et al. Electrocardiographic characteristics at initial diagnosis in patients with isolated left ventricular noncompaction. Am J Cardiol 2009;104:984-9.

76 Corrado D, Basso C, Buja G, et al. Right bundle branch block, right precordial ST-segment elevation, and sudden death in young people. Circulation 2001;103:710-17.

77 Schneider JF, Thomas HE, Kreger BE, et al. Newly acquired right bundle-branch block: the Framingham Study. Ann Intern Med 1980;92:37-44.

78 Brugada J, Brugada R, Antzelevitch C, et al. Long-term follow-up of individuals with the electrocardiographic pattern of right bundle-branch block and ST-segment elevation in precordial leads V1 to V3. Circulation 2002;105:73-8.

79 Steffel J, Kobza R, Namdar M, et al. Electrophysiological findings in patients with isolated left ventricular non-compaction. Europace 2009;11:1193-200.

80 Nelson GS, Curry CW, Wyman BT, et al. Predictors of systolic augmentation from left ventricular preexcitation in patients with dilated cardiomyopathy and intraventricular conduction delay. Circulation 2000;101:2703-9.

81 Xiao HB, Brecker SJ, Gibson DG. Relative effects of left ventricular mass and conduction disturbance on activation in patients with pathological left ventricular hypertrophy. Br Heart J 1994;71:548-53.
82 Baman TS, Lange DC, Ilg KJ, et al. Relationship between burden of premature ventricular complexes and left ventricular function. Heart Rhythm 2010;7:865-9.

83 Braunwald E, Brockenbrough EC, Morrow AG. Hypertrophic subaortic stenosis-a broadened concept. Circulation 1962;26:161-5.

84 Marek JC. Electrocardiography and preparticipation screening of competitive high school athletes. Ann Int Med 2011;153:131-2.

85 Baggish AL, Hutter AM Jr, Wang F, et al. Cardiovascular screening in college athletes with and without electrocardiography: a cross-sectional study. Ann Intern Med 2010;152:269-75.

86 Biffi A, Maron BJ, Verdile L, et al. Impact of physical deconditioning on ventricular tachyarrhythmias in trained athletes. J Am Coll Cardiol 2004;44:1053-8.

87 Biffi A, Pelliccia A, Verdile L, et al. Long-term clinical significance of frequent and complex ventricular tachyarrhythmias in trained athletes. J Am Coll Cardiol 2002:40:446-52.

$88 \mathrm{Kim} \mathrm{JH}$, Noseworthy PA, McCarty D, et al. Significance of electrocardiographic right bundle branch block in trained athletes. Am J Cardiol 2011;107:1083-9.

89 Rich S, Dantzker DR, Ayres SM, et al. Primary pulmonary hypertension. A national prospective study. Ann Intern Med 1987;107:216-23.

90 Bossone $E$, Paciocco $G$, larussi $D$, et al. The prognostic role of the ECG in primary pulmonary hypertension. Chest 2002;121:513-18.

91 Henkens IR, Gan CT, van Wolferen SA, et al. ECG monitoring of treatment response in pulmonary arterial hypertension patients. Chest 2008;134:1250-7.

92 Ahearn GS, Tapson VF, Rebeiz A, et al. Electrocardiography to define clinical status in primary pulmonary hypertension and pulmonary arterial hypertension secondary to collagen vascular disease. Chest 2002;122:524-7. 